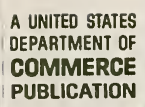

A UNITED STATES DEPARTMENT OF COMMERCE PUBLICATION

\section{NBS SPECIAL PUBLICATION}

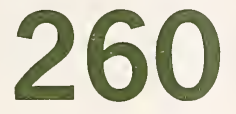

SUPPLEMENT JULY 1971

\title{
Standard Reference Materials
} Price and Availability List

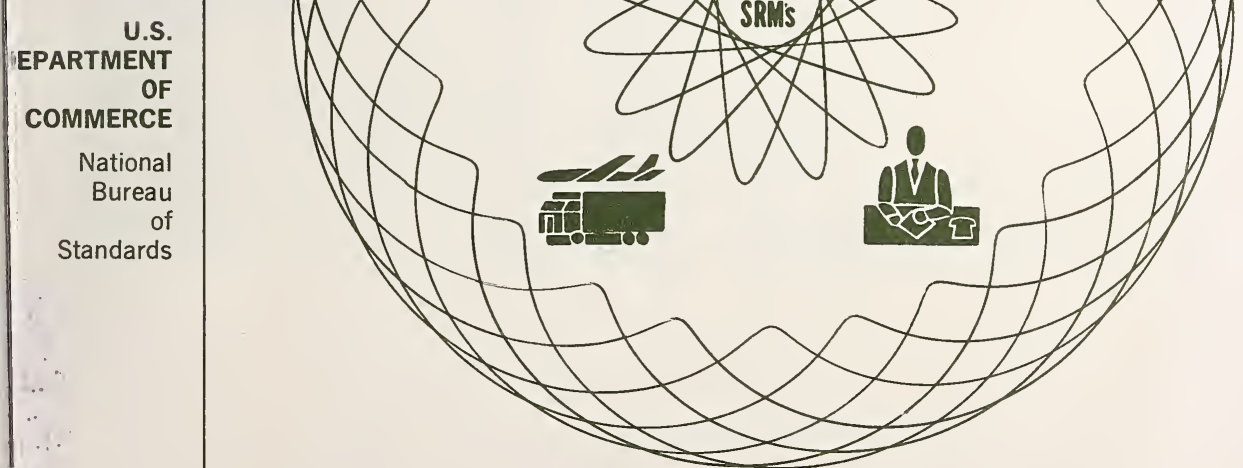




\title{
IMPORTANT NOTICE TO PURCHASERS AND USERS OF NBS STANDARD REFERENCE MATERIALS
}

The Office of Standard Reference Materials no longer issues the Quarterly Insert Sheets to update the current issue of the SRM Catalog. Instead a Standard Reference Material Availability and Price List is issued semiannually. The format has been changed to improve readability and the List is organized as follows:

Section I - A list of all classes of materials currently available arranged by Standard Reference Material (SRM), Research Material (RM), and General Material (GM) numbers, together with type, unit of issue, and current price.

Section II - A short description, arranged by catalog category, of all SRM's issued since the effective date of the current catalog and therefore not contained therein. For ease of reproduction, tables have been condensed and are, in general, not in the same format used in the catalog. (Please note that the values shown are nominal values. The actual values certified are given on the Certificate which accompanies the material.) The unit of issue and price are given after the description of each SRM.

Section III - A list, arranged by SRM, RM, and GM numbers, of all items that have gone out of stock since the effective date of the current catalog. A remarks column gives information concerning alternate SRM's, when the renewal SRM is expected, and similar information.

Section IV - Changes in policy, ordering, shipping, and information of a general nature.

Catalogs are printed without prices to eliminate the need for an annual catalog. New issues of Standard Reference Materials Availability and Price List are mailed automatically to all current customers and those who have completed our Technical Point of Contact Questionnaire.

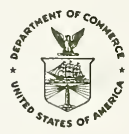

\author{
J. Paul Cali, Chief \\ Office of Standard Reference Materials
}

July 1971

\section{TECHNICAL INQUIRIES}

All technical inquiries regarding SRM's, RM's, and GM's should be directed to the Office of Standard Reference Materials, National Bureau of Standards, Washington, D.C. 20234. Telephone (301) $921-2045$. 
SECTION I

AVAILABILITY AND PRICE LIST

A. STANDARD REFERENCE MATERIALS

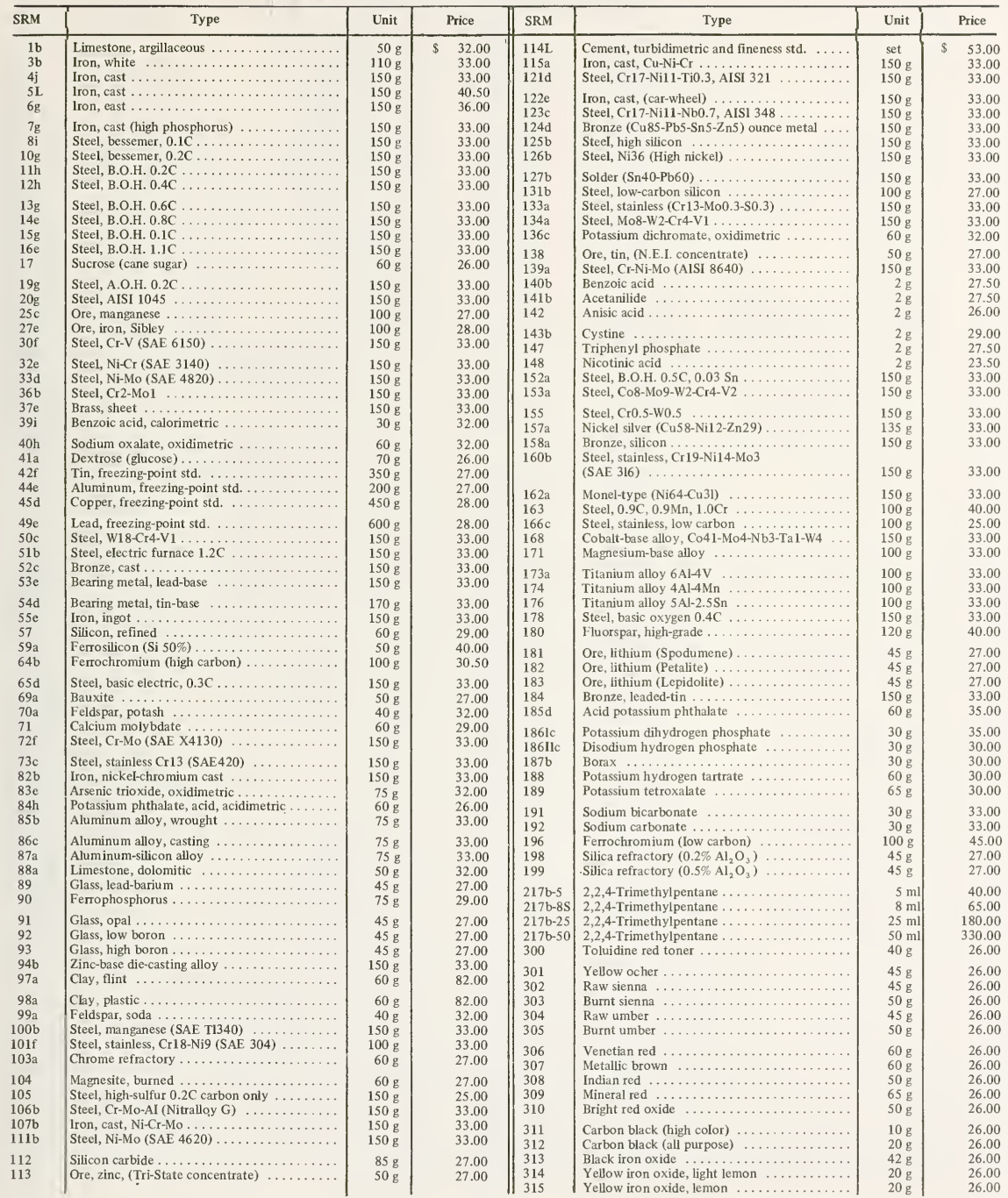




\begin{tabular}{|c|c|c|c|c|c|c|c|}
\hline SRM & Type & Unit & Price & SRM & Type & Unit & Price \\
\hline $\begin{array}{l}316 \\
317 \\
318 \\
319 \\
320\end{array}$ & $\begin{array}{l}\text { Yellow iron oxide, orange } \ldots \ldots \ldots \ldots \ldots \ldots \\
\text { Yellow iron oxide, dark orange } \ldots \ldots \ldots \ldots \\
\text { Lampblack } \ldots \ldots \ldots \ldots \ldots \ldots \ldots \\
\text { Primrose chrome yellow } \ldots \ldots \ldots \ldots \ldots \ldots \ldots \\
\text { Lemon clirome yellow } \ldots \ldots \ldots \ldots \ldots \ldots \ldots\end{array}$ & $\begin{array}{l}25 \mathrm{~g} \\
40 \mathrm{~g} \\
15 \mathrm{~g} \\
65 \mathrm{~g} \\
60 \mathrm{~g}\end{array}$ & $\begin{array}{l}26.00 \\
26.00 \\
26.00 \\
26.00 \\
26.00\end{array}$ & $\begin{array}{l}441 \\
442 \\
443\end{array}$ & 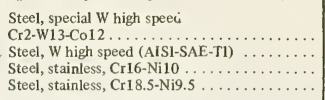 & $\begin{array}{l}\text { ea } \\
\text { ea } \\
\text { ea } \\
\text { ea }\end{array}$ & $\begin{array}{l}35.00 \\
35.00 \\
35.00 \\
35.00\end{array}$ \\
\hline $\begin{array}{l}321 \\
322 \\
323 \\
324 \\
325\end{array}$ & $\begin{array}{l}\text { M edium chrome yellow } \ldots \ldots \ldots \ldots \ldots \ldots \\
\text { Light chrome orange } \ldots \ldots \ldots \ldots \ldots \ldots \ldots \ldots \\
\text { Dark chrome orange } \ldots \ldots \ldots \ldots \ldots \ldots \ldots \ldots \ldots \\
\text { Ultramarine blue } \ldots \ldots \ldots \ldots \ldots \ldots \ldots \ldots \ldots \ldots \\
\text { Iron blue } \ldots \ldots \ldots \ldots \ldots \ldots \ldots \ldots\end{array}$ & $\begin{array}{r}65 \mathrm{~g} \\
100 \mathrm{~g} \\
100 \mathrm{~g} \\
37 \mathrm{~g} \\
25 \mathrm{~g}\end{array}$ & $\begin{array}{l}26.00 \\
26.00 \\
26.00 \\
26.00 \\
26.00\end{array}$ & $\begin{array}{l}444 \\
445\end{array}$ & 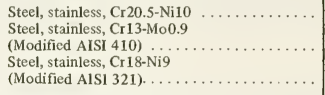 & ea & $\begin{array}{l}35.00 \\
35.00 \\
35.00\end{array}$ \\
\hline $\begin{array}{l}326 \\
327 \\
328 \\
335 \\
337\end{array}$ & $\begin{array}{l}\text { Light chrome green } \ldots \ldots \ldots \ldots \ldots \ldots \ldots \\
\text { Medium clurome green } \ldots \ldots \ldots \ldots \ldots \ldots \\
\text { Dark chrome green } \ldots \ldots \ldots \ldots \ldots \\
\text { Steel, B.O.H. } 0.1 \mathrm{C} \text { (carbon only) } \ldots \ldots \ldots \ldots \\
\text { Steel, B.O.H. } 1.1 \mathrm{C} \text { (carbon only) } \ldots \ldots \ldots \ldots\end{array}$ & $\begin{array}{r}60 \mathrm{~g} \\
50 \mathrm{~g} \\
45 \mathrm{~g} \\
300 \mathrm{~g} \\
300 \mathrm{~g}\end{array}$ & $\begin{array}{l}26.00 \\
26.00 \\
26.00 \\
27.00 \\
27.00\end{array}$ & 448 & 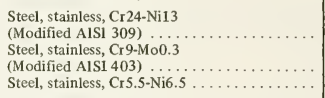 & $\begin{array}{l}\text { ea } \\
\text { ea } \\
\text { ea }\end{array}$ & $\begin{array}{l}35.00 \\
35.00 \\
35.00\end{array}$ \\
\hline $\begin{array}{l}340 \\
341 \\
342 \\
342 a\end{array}$ & 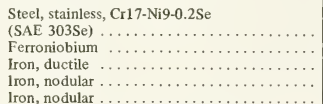 & $\begin{array}{l}150 \mathrm{~g} \\
100 \mathrm{~g} \\
150 \mathrm{~g} \\
150 \mathrm{~g}\end{array}$ & $\begin{array}{l}40.00 \\
45.00 \\
33.00 \\
33.00 \\
35.00\end{array}$ & $\begin{array}{l}450 \\
461 \\
462 \\
463 \\
464\end{array}$ & 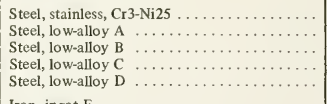 & $\begin{array}{l}\text { ea } \\
\text { ea } \\
\text { ea } \\
\text { ea } \\
\text { ea }\end{array}$ & $\begin{array}{l}35.00 \\
35.00 \\
35.00 \\
35.00 \\
35.00\end{array}$ \\
\hline $\begin{array}{l}343 \\
344 \\
345 \\
346\end{array}$ & 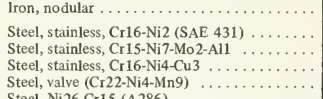 & $\begin{array}{l}150 \mathrm{~g} \\
150 \mathrm{~g} \\
150 \mathrm{~g} \\
150 \mathrm{~g} \\
150 \mathrm{~g}\end{array}$ & $\begin{array}{l}35.00 \\
33.00 \\
33.00 \\
33.00 \\
40.00\end{array}$ & $\begin{array}{l}465 \\
466 \\
467 \\
468 \\
480\end{array}$ & $\begin{array}{l}\text { Iron, ingot } \mathrm{E} \ldots \ldots \ldots \ldots \ldots \ldots \ldots \ldots \ldots \\
\text { Iron, ingot } \mathrm{F} \ldots \ldots \ldots \ldots \ldots \ldots \ldots \ldots \\
\text { Steel, low-alloy } \mathrm{G} \ldots \ldots \ldots \ldots \ldots \ldots \ldots \ldots \\
\text { Steel, low-alloy } \mathrm{H} \ldots \ldots \ldots \ldots \ldots \ldots \\
\text { Microprobe, Tungsten }-20 \% \text { Molybdenum alloy }\end{array}$ & $\begin{array}{l}\text { ea } \\
\text { ea } \\
\text { ea } \\
\text { ea } \\
\text { ea }\end{array}$ & $\begin{array}{r}35.00 \\
35.00 \\
35.00 \\
35.00 \\
125.00\end{array}$ \\
\hline $\begin{array}{l}348 \\
349 \\
350 \\
352 \\
353\end{array}$ & $\begin{array}{l}\text { Steel, Ni26-Cr15 (A286) ............. } \\
\text { Nickel-base alloy (Ni57-Col4-Cr20) } \ldots \ldots \ldots \\
\text { Benzoic acid, acidimetric } \ldots \ldots \ldots \ldots \\
\text { Titanium, unalloyed, for hydrogen } \ldots \ldots \ldots \ldots \\
\text { Titanium, unalloyed, for hydrogen } \ldots \ldots \ldots \ldots\end{array}$ & $\begin{array}{r}150 \mathrm{~g} \\
150 \mathrm{~g} \\
30 \mathrm{~g} \\
20 \mathrm{~g} \\
20 \mathrm{~g}\end{array}$ & $\begin{array}{l}33.00 \\
33.00 \\
26.00 \\
35.00 \\
35.00\end{array}$ & $\begin{array}{l}481 \\
482 \\
483 \\
485 \\
493\end{array}$ & $\begin{array}{l}\text { Microprobe, Gold-silver wires } \ldots \ldots \ldots \ldots \ldots \\
\text { Microprobe, Gold-copper wires } \ldots \ldots \ldots \ldots \ldots \\
\text { Microprobe, Iron-3\% silicon } \ldots \ldots \ldots \ldots \ldots \ldots \\
\text { Austenite in ferrite } \ldots \ldots \ldots \ldots \ldots \ldots \ldots \\
\text { Iron carbide in ferrite } \ldots \ldots \ldots \ldots \ldots \ldots \ldots\end{array}$ & $\begin{array}{l}\text { set } \\
\text { set } \\
\text { ea } \\
\text { ea } \\
\text { ea }\end{array}$ & $\begin{array}{r}130.00 \\
130.00 \\
50.00 \\
85.00 \\
85.00\end{array}$ \\
\hline $\begin{array}{l}355 \\
356 \\
360 \mathrm{a} \\
361 \\
362\end{array}$ & $\begin{array}{l}\text { Titanium, unalloyed, for oxygen } \ldots \ldots \ldots \ldots \\
\text { Titanium alloy, } 6 \mathrm{Al}-4 \mathrm{~V} \ldots \ldots \ldots \ldots \ldots \\
\text { Zircaloy-2 } \ldots \ldots \ldots \\
\text { Steel, AlSI } 4340 \text {, chip } \ldots \ldots \ldots \ldots \ldots \ldots \\
\text { Steel }\end{array}$ & $\begin{array}{r}20 \mathrm{~g} \\
20 \mathrm{~g} \\
100 \mathrm{~g} \\
150 \mathrm{~g}\end{array}$ & $\begin{array}{l}40.00 \\
40.00 \\
55.00 \\
33.00\end{array}$ & $\begin{array}{l}592 \\
593 \\
594 \\
595 \\
596\end{array}$ & $\begin{array}{l}\text { Hydrocarbon blends - Blend No. } 1 \ldots \ldots \ldots \\
\text { Hydrocarbon blends - Blend No. } 2 \ldots \ldots \ldots \\
\text { Hydrocarbon blends - Blend No. } 3 \ldots \ldots \\
\text { Hydrocarbon blends - Blend No. } 4 \ldots \ldots \ldots \\
\text { Hydrocarbon blends - Blend No. } 5 \quad \ldots \ldots\end{array}$ & $\begin{array}{l}\text { set } \\
\text { set } \\
\text { set } \\
\text { set } \\
\text { set }\end{array}$ & $\begin{array}{l}32.00 \\
32.00 \\
32.00 \\
32.00 \\
32.00\end{array}$ \\
\hline $\begin{array}{l}363 \\
364 \\
365 \\
366\end{array}$ & $\begin{array}{l}\text { Steel, AIS1 94B17 (modified), chip } \ldots \ldots \ldots \\
\text { Steel, Cr-V (modified), chip ........... } \\
\text { Steel, high carbon (modified), chip } \ldots \ldots \ldots \\
\text { Iron, electrolytic, chip } \ldots \ldots \ldots \ldots \\
\text { Set } 1 \text { ea of } 361,362,363,364 \text { and } 365 \ldots \ldots\end{array}$ & $\begin{array}{l}150 \mathrm{~g} \\
150 \mathrm{~g} \\
150 \mathrm{~g} \\
150 \mathrm{~g} \\
\text { set }\end{array}$ & $\begin{array}{r}33.00 \\
33.00 \\
33.00 \\
100.00\end{array}$ & $\begin{array}{l}597 \\
598 \\
599 \\
610 \\
611\end{array}$ & $\begin{array}{l}\text { Hydrocarbon blends - Blend No. } 6 \ldots \ldots \ldots \\
\text { Hydrocarbon blends - Blend No. } 7 \ldots \ldots \ldots \\
\text { Hydrocarbon blends - Blend No. } 8 \ldots \ldots \\
\text { Glass, trace elements } 500 \mathrm{ppm}, 3 \mathrm{~mm} \\
\text { Glass, trace elements } 500 \mathrm{ppm}, 1 \mathrm{~mm}\end{array}$ & $\begin{array}{l}\text { set } \\
\text { set } \\
\text { set } \\
\text { ea } \\
\text { ea }\end{array}$ & $\begin{array}{l}32.00 \\
32.00 \\
32.00 \\
50.00 \\
50.00\end{array}$ \\
\hline $370 \mathrm{~d}$ & Zinc oxide (Set of 4$) \ldots \ldots \ldots \ldots \ldots \ldots$ & $8 \mathrm{~kg}$ & 33.80 & $\begin{array}{l}612 \\
613\end{array}$ & Glass, trace elements $50 \mathrm{ppm}, 3 \mathrm{~mm} \ldots \ldots \ldots$ & ea & $\begin{array}{l}50.00 \\
50.00\end{array}$ \\
\hline $\begin{array}{l}371 \mathrm{f} \\
372 \mathrm{~g} \\
373 \mathrm{f} \\
374 \mathrm{c}\end{array}$ & $\begin{array}{l}\text { Sulfur (Set of } 4) \ldots \ldots \ldots \ldots \\
\text { Stearic acid (Set of } 4 \text { ) } \ldots \ldots \ldots \ldots \ldots \\
\text { Benzothiazyl disulfide }(\text { Set of } 4) \ldots \ldots \ldots \ldots \\
\text { Tetramethylthiuram disulfide } \ldots \ldots \ldots\end{array}$ & $\begin{array}{r}6 \mathrm{~kg} \\
3.2 \mathrm{~kg} \\
2 \mathrm{~kg} \\
2 \mathrm{~kg}\end{array}$ & $\begin{array}{l}38.00 \\
31.00 \\
40.00 \\
40.00\end{array}$ & $\begin{array}{l}613 \\
614 \\
615 \\
616\end{array}$ & $\begin{array}{l}\text { Glass, trace elements } 50 \mathrm{ppm}, 1 \mathrm{~mm} \ldots \ldots \\
\text { Glass, trace elements } 1 \mathrm{ppm}, 3 \mathrm{~mm} \ldots \ldots \\
\text { Glass, trace elements } 1 \mathrm{ppm}, 1 \mathrm{~mm} \ldots \ldots \\
\text { Glass, trace elements } .02 \mathrm{ppm}, 3 \mathrm{~mm} \ldots \ldots\end{array}$ & $\begin{array}{l}\text { ea } \\
\text { ea } \\
\text { ea } \\
\text { ea }\end{array}$ & $\begin{array}{l}50.00 \\
50.00 \\
50.00 \\
50.00\end{array}$ \\
\hline $\begin{array}{l}375 \mathrm{f} \\
376 \mathrm{a} \\
377 \\
378 \mathrm{a} \\
379 \\
380\end{array}$ & $\begin{array}{l}\text { Channel black (Set of } 4) \ldots \ldots \ldots \ldots \ldots \\
\text { Light magnesia } \ldots \ldots \ldots \ldots \\
\text { Phenyl-beta-naphthylamine } \ldots \ldots \ldots \ldots \ldots \ldots \\
\text { Oil furnace black }(\text { Set of } 4) \ldots \ldots \ldots \ldots \ldots \\
\text { Conducting black } \ldots \ldots \ldots \ldots \ldots \ldots \ldots \ldots \\
\text { Calcium carbonate } \ldots \ldots \ldots \ldots \ldots \ldots \ldots\end{array}$ & $\begin{array}{r}28 \mathrm{~kg} \\
450 \mathrm{~g} \\
600 \mathrm{~g} \\
28 \mathrm{~kg} \\
5.5 \mathrm{~kg} \\
6 \mathrm{~kg}\end{array}$ & $\begin{array}{l}67.00 \\
25.25 \\
26.75 \\
36.00 \\
26.25 \\
25.25\end{array}$ & $\begin{array}{l}617 \\
618 \\
619 \\
625 \\
626 \\
627\end{array}$ & 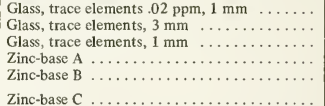 & $\begin{array}{l}\text { ea } \\
\text { set } \\
\text { set } \\
\text { ea } \\
\text { ea }\end{array}$ & $\begin{array}{r}50.00 \\
150.00 \\
150.00 \\
50.00 \\
50.00\end{array}$ \\
\hline $\begin{array}{l}381 \\
382 \mathrm{a} \\
383 \\
384\end{array}$ & 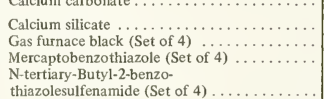 & $\begin{array}{r}6 \mathrm{~kg} \\
4 \mathrm{~kg} \\
32 \mathrm{~kg} \\
3.2 \mathrm{~kg}\end{array}$ & $\begin{array}{l}25.25 \\
25.25 \\
52.00 \\
33.00\end{array}$ & $\begin{array}{l}627 \\
628 \\
629 \\
630 \\
631\end{array}$ & 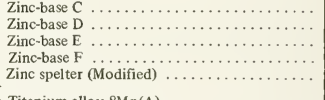 & $\begin{array}{l}\text { ea } \\
\text { ea } \\
\text { ea } \\
\text { ea } \\
\text { ea }\end{array}$ & $\begin{array}{l}50.00 \\
50.00 \\
50.00 \\
50.00 \\
50.00\end{array}$ \\
\hline $385 b$ & $\begin{array}{l}\text { thiazolesulfenamide }(\text { Set of } 4) \ldots \ldots \ldots \ldots \\
\text { Natural rubber } \ldots \ldots \ldots \ldots \ldots \ldots \ldots\end{array}$ & $\begin{array}{r}3.2 \mathrm{~kg} \\
31.4 \mathrm{~kg}\end{array}$ & $\begin{array}{r}37.00 \\
105.00\end{array}$ & 641 & $\begin{array}{l}\text { Titanium alloy } 8 \mathrm{Mn}(\mathrm{A}) \ldots \ldots \ldots \ldots \ldots \ldots \\
\text { Titanium alloy } 8 \mathrm{Mn}(\mathrm{B}) \quad \ldots \ldots \ldots \ldots \ldots\end{array}$ & $\begin{array}{l}\text { ea } \\
\text { ea }\end{array}$ & $\begin{array}{l}50.00 \\
50.00\end{array}$ \\
\hline $\begin{array}{l}386 \mathrm{~g} \\
388 \mathrm{e} \\
389 \\
391\end{array}$ & $\begin{array}{l}\text { Styrene-butadiene type } 1500 \ldots \ldots \ldots \ldots \\
\text { Butyl rubber } \ldots \ldots \ldots \\
\text { Styrene-butadiene, type } 1503 \ldots \ldots \ldots \\
\text { Acrylonitrile-butadiene rubber } \ldots \ldots \ldots \ldots\end{array}$ & $\begin{array}{l}34 \mathrm{~kg} \\
37 \mathrm{~kg} \\
34 \mathrm{~kg} \\
25 \mathrm{~kg}\end{array}$ & $\begin{array}{r}67.00 \\
105.00 \\
54.00 \\
105.00\end{array}$ & $\begin{array}{l}643 \\
644 \\
645\end{array}$ & $\begin{array}{l}\text { Titanium alloy } 8 \mathrm{Mn}(\mathrm{C}) \ldots \ldots \ldots \ldots \\
\text { Titanium alloy } 2 \mathrm{Cr}-2 \mathrm{Fe}-2 \mathrm{Mo}(\mathrm{A}) \\
\text { Titanium alloy } 2 \mathrm{Cr}-2 \mathrm{Fe}-2 \mathrm{Mo}(\mathrm{B}) \ldots \ldots \ldots\end{array}$ & $\begin{array}{l}\text { ea } \\
\text { ea } \\
\text { ea }\end{array}$ & $\begin{array}{l}50.00 \\
50.00 \\
50.00\end{array}$ \\
\hline $404 \mathrm{a}$ & $\begin{array}{l}\text { Acrylonitrile-butadiene rubber } \ldots \ldots \ldots \ldots \ldots \\
\text { Steel, basic electric } \ldots \ldots \ldots \ldots \ldots \ldots \ldots\end{array}$ & $\begin{array}{l}25 \mathrm{~kg} \\
\text { ea }\end{array}$ & $\begin{array}{r}105.00 \\
30.00\end{array}$ & $\begin{array}{l}646 \\
654 a\end{array}$ & $\begin{array}{l}\text { Titanium alloy } 2 \mathrm{Cr}-2 \mathrm{Fe}-2 \mathrm{Mo}(\mathrm{C}) \ldots \ldots \ldots \ldots \\
\text { Titanium alloy, } 6 \mathrm{Al}-4 \mathrm{~V} \ldots \ldots \ldots \ldots \ldots\end{array}$ & $\begin{array}{l}\text { ea } \\
\text { ea }\end{array}$ & $\begin{array}{l}50.00 \\
35.00\end{array}$ \\
\hline $\begin{array}{l}405 \mathrm{a} \\
407 \mathrm{a} \\
408 \mathrm{a} \\
409 \mathrm{~b} \\
413\end{array}$ & $\begin{array}{l}\text { Steel, medium manganese } \ldots \ldots \ldots \ldots \ldots \ldots \ldots \\
\text { Steel, chromium-yanadium } \ldots \ldots \ldots \ldots \ldots \ldots \\
\text { Steel, chromium-nickel } \ldots \ldots \ldots \ldots \ldots \ldots \ldots \\
\text { Steel, nickel } \ldots \ldots \ldots \ldots \ldots \ldots \ldots \ldots \ldots \ldots \\
\text { Steel, A.O.H. } 0.4 \text { C } \ldots \ldots \ldots \ldots \ldots \ldots \ldots\end{array}$ & $\begin{array}{l}\text { ea } \\
\text { ea } \\
\text { ea } \\
\text { ea } \\
\text { ea }\end{array}$ & $\begin{array}{l}30.00 \\
30.00 \\
30.00 \\
30.00 \\
30.00\end{array}$ & $\begin{array}{l}661 \\
662 \\
663\end{array}$ & $\begin{array}{l}\text { Steel, AISI 4340, rod } \\
\text { (Sold in sets only-666, 668) } \ldots \ldots \ldots \ldots \ldots \\
\text { Steel, AISI 94B17 (modified), rod } \\
\text { (Sold in sets only-667,668) } \ldots \ldots \ldots \ldots \ldots \\
\text { Steel, Cr-V (modified), rod }\end{array}$ & & \\
\hline $\begin{array}{l}414 \\
417 \mathrm{a} \\
418 \\
420 \mathrm{a} \\
427\end{array}$ & $\begin{array}{l}\text { Steel, Cr-Mo (SAE } 4140) \ldots \ldots \ldots \ldots \\
\text { Steel, B.O.H. } 0.4 \mathrm{C} \ldots \ldots \ldots \\
\text { Steel, Cr-Mo }(\text { SAE X4130) } \ldots \ldots \ldots \ldots \\
\text { Iron, ingot } \ldots \ldots \ldots \ldots \\
\text { Steel, Cr-Mo (boron only) (SAE } 4 \text { (Sis0) } \ldots \ldots\end{array}$ & $\begin{array}{l}\text { ea } \\
\text { ea } \\
\text { ea } \\
\text { ea } \\
\text { ea }\end{array}$ & $\begin{array}{l}30.00 \\
30.00 \\
30.00 \\
30.00 \\
30.00\end{array}$ & 664 & $\begin{array}{l}\text { (Sold in sets only }-667,668) \ldots \ldots \ldots \ldots \\
\text { Steel, high carbon (modified), rod } \\
\text { (Sold singly and in sets }-668) \ldots \ldots \ldots \ldots \ldots \\
\text { Iron, electrolytic, rod } \\
\text { (Sold in sets only }-666,668) \ldots \ldots \ldots \ldots\end{array}$ & ea & 25.00 \\
\hline $\begin{array}{l}432 \\
436 \\
437 \\
438 \\
439\end{array}$ & $\begin{array}{l}\text { Tin B } \ldots \ldots \\
\text { Steel, special Cr6-Mo3-W10 } \ldots \ldots \ldots \ldots \ldots \\
\text { Steel, special Cr8-Mo2-W3-Co } 3 \ldots \ldots \ldots \\
\text { Steel, Mo high speed (AISI-SAE-M30) } \ldots \ldots \ldots \\
\text { Steel, Mo high speed (AISI-SAE-M } 36 \text { ) } \ldots \ldots \ldots\end{array}$ & $\begin{array}{l}\text { ea } \\
\text { ea } \\
\text { ea } \\
\text { ea } \\
\text { ea }\end{array}$ & $\begin{array}{l}35.00 \\
35.00 \\
35.00 \\
35.00 \\
35.00\end{array}$ & $\begin{array}{l}666 \\
667 \\
668 \\
\\
671 \\
672\end{array}$ & 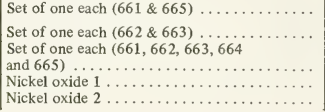 & $\begin{array}{l}\text { set } \\
\text { set } \\
\text { set } \\
25 \mathrm{~g} \\
25 \mathrm{~g}\end{array}$ & $\begin{array}{l}40.00 \\
40.00 \\
75.00 \\
35.00 \\
35.00\end{array}$ \\
\hline
\end{tabular}




\begin{tabular}{|c|c|c|c|c|c|c|c|}
\hline SRM & Type & Unit & Price & SRM & Type & Unit & Price \\
\hline $\begin{array}{l}673 \\
680 \mathrm{~L}-1 \\
680 \mathrm{~L}-2 \\
681 \mathrm{~L}-1 \\
681 \mathrm{~L}-2\end{array}$ & $\begin{array}{l}\text { Nickel oxide } 3 \ldots \ldots \ldots \ldots \\
\text { Platinum, high-purity } \ldots \ldots \ldots \ldots \ldots \\
\text { Platinum, high-purity } \ldots \ldots \ldots \ldots \ldots \ldots \\
\text { Platinum, doped } \ldots \ldots \ldots \ldots \ldots \ldots \\
\text { Platinum, doped } \ldots \ldots \ldots \ldots \ldots \ldots\end{array}$ & $\begin{array}{l}25 \mathrm{~g} \\
\text { ea } \\
\text { ea } \\
\text { ea } \\
\text { ea }\end{array}$ & $\begin{array}{r}\$ 35.00 \\
40.00 \\
190.00 \\
40.00 \\
190.00\end{array}$ & $\begin{array}{r}\text { D } 841 \\
845 \\
\text { D845 } \\
846 \\
\text { D846 }\end{array}$ & $\begin{array}{l}\text { Steel, W high speed (AIS1-SAE-T1) } \ldots \ldots \ldots \ldots \\
\text { Steel, Cr13-Mo0.9 (Modified AlS1 } 410 \text { ) } \ldots \ldots \\
\text { Steel, Cr13-Mo0.9 (Modified AlSI } 410 \text { ) } \ldots \ldots \\
\text { Steel, Cr18-Ni9 (Modified A1S1 321) } \ldots \ldots \ldots \\
\text { Steel, Cr18-Ni9 (Modified A1SI 321) } \ldots \ldots \ldots\end{array}$ & $\begin{array}{l}\text { ea } \\
\text { ea } \\
\text { ea } \\
\text { ea } \\
\text { ea }\end{array}$ & $\begin{array}{l}50.00 \\
42.50 \\
50.00 \\
42.50 \\
50.00\end{array}$ \\
\hline $\begin{array}{l}682 \\
683 \\
685-\mathrm{R} \\
685-\mathrm{W} \\
700 \mathrm{~b}\end{array}$ & $\begin{array}{l}\text { Zinc, high-purity } \ldots \ldots \ldots \ldots \ldots \ldots \ldots \ldots \\
\text { Zinc metal } \ldots \ldots \ldots \ldots \ldots \\
\text { Gold, high-purity (rod) } \ldots \ldots \ldots \ldots \ldots \ldots \\
\text { Gold, high-purity (wire) } \ldots \ldots \ldots \ldots \ldots \\
\text { Paper, light-sensitive } \ldots \ldots \ldots \ldots \ldots \ldots \ldots\end{array}$ & $\begin{array}{r}\text { ea } \\
\text { ea } \\
\text { ea } \\
\text { ea } \\
\text { pkg }\end{array}$ & $\begin{array}{l}90.00 \\
55.00 \\
55.00 \\
55.00 \\
40.00\end{array}$ & $\begin{array}{r}\text { D847 } \\
\text { D } 848 \\
849 \\
\text { D } 849 \\
850\end{array}$ & $\begin{array}{l}\text { Steel, Cr24-Ni13 (Modified AISI 309) } \ldots \ldots \ldots \\
\text { Steel, Cr9-Mo0.3 (Modified AISI 403) } \ldots \ldots \ldots \\
\text { Steel, Cr5.5-Ni6.5 } \ldots \ldots \ldots \ldots \ldots \ldots \ldots \ldots \ldots \\
\text { Steel, Cr5.5-Ni6.5 } \ldots \ldots \ldots \ldots \ldots \ldots \ldots \ldots \ldots \\
\text { Steel, Cr3-Ni25 } \ldots \ldots \ldots \ldots \ldots \ldots \ldots \ldots \ldots\end{array}$ & $\begin{array}{l}\text { ea } \\
\text { ea } \\
\text { ea } \\
\text { ea } \\
\text { ea }\end{array}$ & $\begin{array}{l}50.00 \\
50.00 \\
42.50 \\
50.00 \\
42.50\end{array}$ \\
\hline $\begin{array}{l}701 \mathrm{~b} \\
702 \\
703 \\
704 \mathrm{a} \\
705\end{array}$ & $\begin{array}{l}\text { Paper, standard faded strips } \ldots \ldots \ldots \ldots \ldots \ldots \\
\text { Plastic chips, light-sensitive } \ldots \ldots \ldots \ldots \ldots \ldots \\
\text { Plastic chips, light-sensitive } \ldots \ldots \ldots \ldots \ldots \ldots \\
\text { Paper, internal tearing resistance } \ldots \ldots \ldots \ldots \\
\text { Polystyrene, narrow molecular weight } \ldots \ldots \ldots\end{array}$ & $\begin{array}{l}\text { bklt } \\
\text { pkg } \\
\text { pkg } \\
\text { set (4) } \\
2 \mathrm{~g}\end{array}$ & $\begin{array}{r}155.00 \\
40.00 \\
40.00 \\
56.20 \\
33.00\end{array}$ & $\begin{array}{r}\text { D850 } \\
911 \\
912 \\
913 \\
914\end{array}$ & 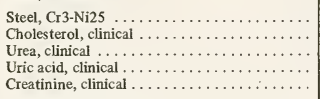 & $\begin{array}{l}\text { ea } \\
0.5 \mathrm{~g} \\
25 \mathrm{~g} \\
10 \mathrm{~g} \\
10 \mathrm{~g}\end{array}$ & $\begin{array}{l}50.00 \\
30.00 \\
36.00 \\
30.00 \\
36.00\end{array}$ \\
\hline $\begin{array}{l}706 \\
710 \\
711 \\
712 \\
713\end{array}$ & $\begin{array}{l}\text { Polystyrene, broad molecular weight } \ldots \ldots \ldots \\
\text { Glass, soda-lime silica } \ldots \ldots \ldots \ldots \ldots \ldots \\
\text { Glass, lead-silica } \ldots \ldots \ldots \ldots \\
\text { Glass, mixed alkali lead silicate } \ldots \ldots \ldots \ldots \ldots \\
\text { Glass, dense barium crown } \ldots \ldots \ldots \ldots \ldots\end{array}$ & $\begin{array}{r}18 \mathrm{~g} \\
2 \mathrm{lb} \\
3 \mathrm{lb} \\
0.5 \mathrm{lb} \\
0.5 \mathrm{lb}\end{array}$ & $\begin{array}{l}33.00 \\
52.00 \\
75.00 \\
38.00 \\
38.00\end{array}$ & $\begin{array}{l}915 \\
916 \\
917 \\
918 \\
922\end{array}$ & $\begin{array}{l}\text { Calcium carbona te, clinical } \ldots \ldots \ldots \ldots \ldots \ldots \\
\text { Bilirubin, clinical } \ldots \ldots \ldots \ldots \ldots \ldots \ldots \ldots \\
\text { D-Glucose, clinical } \ldots \ldots \ldots \ldots \ldots \ldots \\
\text { Potassium chloride, clinical } \ldots \ldots \ldots \ldots \ldots \ldots \\
\text { Tris(hy droxymethyl)aminomethane }\end{array}$ & $\begin{array}{l}20 \mathrm{~g} \\
100 \mathrm{mg} \\
25 \mathrm{~g} \\
30 \mathrm{~g}\end{array}$ & $\begin{array}{l}30.00 \\
92.00 \\
43.00 \\
40.00\end{array}$ \\
\hline $\begin{array}{l}714 \\
715 \\
716 \\
717 \\
720\end{array}$ & $\begin{array}{l}\text { Glass, alkaline earth alumina silicate } \ldots \ldots \ldots \\
\text { Glass, alkali-free aluminosilicate } \ldots \ldots \ldots \ldots \\
\text { Glass, neutral (borosilicate) } \ldots \ldots \ldots \ldots \ldots \\
\text { Glass, standard, borosilicate } \ldots \ldots \ldots \ldots \\
\text { Sapphire, synthetic }\left(\mathrm{AI}_{2} \mathrm{O}_{3}\right) \ldots \ldots \ldots \ldots \ldots\end{array}$ & $\begin{array}{r}0.5 \mathrm{lb} \\
200 \mathrm{~g} \\
250 \mathrm{~g} \\
1 \mathrm{lb} \\
15 \mathrm{~g}\end{array}$ & $\begin{array}{l}38.00 \\
38.00 \\
38.00 \\
71.00 \\
56.00\end{array}$ & 923 & $\begin{array}{l}\text { Tris(hy droxy methyl)aminomethane } \\
\text { hydrochloride, clinical } \ldots \ldots \ldots \ldots \ldots \ldots \ldots \ldots \\
\text { Glass filters for spectrophotometry, } \\
\text { clinical } \ldots \ldots \ldots \ldots \ldots \ldots \ldots \ldots \ldots \ldots \ldots\end{array}$ & $35 \mathrm{~g}$ & 40.00 \\
\hline $\begin{array}{l}723 \\
724\end{array}$ & $\begin{array}{l}\text { Tris(hydroxy methyl)aminomethane, basimetric } \\
\text { Tris(hy droxy methyl)aminomethane, calorimetric }\end{array}$ & $\begin{array}{l}50 \mathrm{~g} \\
50 \mathrm{~g}\end{array}$ & $\begin{array}{l}50.75 \\
40.00\end{array}$ & $\begin{array}{l}944 \\
945\end{array}$ & $\begin{array}{l}\text { Plutonium sulfate tetrahydrate assay } \ldots \ldots \ldots \\
\text { Plutonium metal, std matrix } \ldots \ldots \ldots \ldots \ldots\end{array}$ & $\begin{array}{r}0.5 \mathrm{~g} \\
5 \mathrm{~g}\end{array}$ & $\begin{array}{r}76.00 \\
500.00\end{array}$ \\
\hline $\begin{array}{l}725 \\
726\end{array}$ & $\begin{array}{l}\text { Mossbauer Differential Chemical Shift } \ldots \ldots \ldots \\
\text { Selenium } \ldots \ldots \ldots \ldots \ldots \ldots \ldots \ldots\end{array}$ & $1 \mathrm{lb}$ & $\begin{array}{r}155.00 \\
45.00\end{array}$ & $\begin{array}{l}948 \\
949 \mathrm{c}\end{array}$ & $\begin{array}{l}\text { Plutonium sulfate hydrate } \ldots \ldots \ldots \ldots \ldots \ldots \\
\text { Plutonium metal assay } \ldots \ldots \ldots \ldots \ldots \ldots\end{array}$ & $\begin{array}{r}0.25 \mathrm{~g} \\
0.5 \mathrm{~g}\end{array}$ & $\begin{array}{r}66.50 \\
123.00\end{array}$ \\
\hline $\begin{array}{l}728 \\
734 S\end{array}$ & $\begin{array}{l}\text { Zinc } \ldots \ldots \ldots \ldots \ldots \ldots \ldots \ldots \\
\text { lron, electrolytic, thermal conductivity, } \\
\text { rod } 6.4 \mathrm{~mm} \text { dia., } 305 \mathrm{~mm} \text { long } \ldots \ldots \ldots \ldots \ldots\end{array}$ & $\begin{array}{l}450 \mathrm{~g} \\
\text { ea }\end{array}$ & $\begin{array}{l}43.00 \\
75.00\end{array}$ & $\begin{array}{l}950 \mathrm{a} \\
951 \\
952\end{array}$ & $\begin{array}{l}\text { Uranium oxide }\left(\mathrm{U}_{3} \mathrm{0}_{s}\right) \ldots \ldots \ldots \ldots \ldots \ldots \\
\text { Boric acid } \ldots \ldots \ldots \ldots \ldots \ldots \ldots \\
\text { Boric acid, } 95 \% \text { enriched }{ }^{\mathrm{i}}{ }_{\mathrm{o}}{ }_{\mathrm{B}} \ldots \ldots \ldots \ldots \ldots \ldots\end{array}$ & $\begin{array}{r}25 \mathrm{~g} \\
100 \mathrm{~g} \\
0.25 \mathrm{~g}\end{array}$ & $\begin{array}{l}28.25 \\
55.00 \\
40.00\end{array}$ \\
\hline $\begin{array}{l}734 \mathrm{~L} 1 \\
734 \mathrm{~L} 2\end{array}$ & $\begin{array}{l}\text { Iron, electrolytic, thermal conductivity, } \\
\text { rod, } 31.8 \mathrm{~mm} \text { dia., } 152 \mathrm{~mm} \text { long } \ldots \ldots \ldots \ldots \\
\text { Iron, electrolytic, thermal conductivity, } \\
\text { rod } 31.8 \mathrm{~mm} \text { dia., } 305 \mathrm{~mm} \text { long } \ldots \ldots \ldots \ldots \\
\text { Copper, thermal expansion, } 2 \text { in. } \ldots \ldots \ldots \ldots\end{array}$ & $\begin{array}{l}\text { ea } \\
\text { ea } \\
\text { ea }\end{array}$ & $\begin{array}{r}85.00 \\
150.00 \\
71.00\end{array}$ & $\begin{array}{l}953 \\
975 \\
976 \\
977 \\
978\end{array}$ & $\begin{array}{l}\text { Neutron density monitor wire } \ldots \ldots \ldots \ldots \ldots \\
\text { Sodium chloride - isotopic } \ldots \ldots \ldots \ldots \ldots \\
\text { Copper metal - isotopic } \ldots \ldots \ldots \ldots \ldots \ldots \ldots \\
\text { Sodjum bromide - isotopic } \ldots \ldots \ldots \ldots \ldots \\
\text { Silver nitrate - isotopic } \ldots \ldots \ldots \ldots \ldots \ldots\end{array}$ & $\begin{array}{l}\text { ea } \\
0.25 \mathrm{~g} \\
0.25 \mathrm{~g} \\
0.25 \mathrm{~g} \\
0.25 \mathrm{~g}\end{array}$ & $\begin{array}{l}35.00 \\
40.00 \\
40.00 \\
40.00 \\
40.00\end{array}$ \\
\hline $\begin{array}{l}736 \mathrm{~L} 2 \\
736 \mathrm{~L} 3 \\
739 \mathrm{LL} 1 \\
739 \mathrm{~L} 2\end{array}$ & $\begin{array}{l}\text { Copper, thermal expansion, } 4 \text { in. . . . } \ldots \ldots \ldots \\
\text { Copper, thermal expansion, } 6 \text { in. } \ldots \ldots \ldots \ldots \\
\text { Fused-silica, thermal expansion, } 2 \text { in. } \ldots \ldots \ldots \\
\text { Fused-silica, thermal expansion, } 4 \text { in. } \ldots \ldots \ldots\end{array}$ & $\begin{array}{l}\text { ea } \\
\text { ea } \\
\text { ea } \\
\text { ea }\end{array}$ & $\begin{array}{r}119.00 \\
167.00 \\
71.00 \\
119.00\end{array}$ & $\begin{array}{l}979 \\
980 \\
981-3 \\
984\end{array}$ & $\begin{array}{l}\text { Chromium nitrate - isotopic } \ldots \ldots \ldots \ldots \ldots \\
\text { Magnesium metal - isotopic } \ldots \ldots \ldots \ldots \ldots \\
\text { Lead - isotopic } \ldots \ldots \ldots \ldots \ldots \ldots \\
\text { Rubidium chloride, isotopic } \ldots \ldots \ldots \ldots \ldots\end{array}$ & $\begin{array}{l}0.25 \mathrm{~g} \\
0.25 \mathrm{~g} \\
\text { set } \\
1 \mathrm{~g}\end{array}$ & $\begin{array}{r}40.00 \\
40.00 \\
105.00 \\
43.00\end{array}$ \\
\hline $\begin{array}{l}739 L 3 \\
740 \\
742 \\
745 \\
746 \\
748\end{array}$ & $\begin{array}{l}\text { Fused-silica, thermal expansion, } 6 \text { in. } \ldots \ldots \ldots \\
\text { Zinc, freezing-point std. } \ldots \ldots \ldots \ldots \ldots \ldots \\
\text { Alumina, high temperature melting point } \ldots \ldots \\
\text { Gold, vapor pressure std. } \ldots \ldots \ldots \ldots \ldots \ldots \\
\text { Cadmium, vapor pressure std. } \ldots \ldots \ldots \ldots \ldots \\
\text { Silver, vapor pressure std. } \ldots \ldots \ldots \ldots \ldots\end{array}$ & $\begin{array}{l}\text { ea } \\
350 \mathrm{~g} \\
10 \mathrm{~g} \\
\text { ea } \\
\text { ea }\end{array}$ & $\begin{array}{r}167.00 \\
70.00 \\
62.50 \\
85.00 \\
65.00\end{array}$ & $\begin{array}{l}1000 \\
1002 b \\
1003 \\
1010 a \\
1011 \\
1013\end{array}$ & $\begin{array}{l}\text { Enameled iron plaques } \ldots \ldots \ldots \ldots \ldots \ldots \ldots \\
\text { Hardboard sheet, } 4 \text { specimens } \ldots \ldots \ldots \ldots \ldots \\
\text { Glass spheres }(5-30 \mu \mathrm{m}) \quad \ldots \ldots \ldots \ldots \ldots \ldots \\
\text { Microcopy test chart } \ldots \ldots \ldots \ldots \ldots \ldots \ldots \ldots \\
\text { Cement, Portland } \ldots \ldots \ldots \ldots \ldots \ldots \ldots \ldots \\
\text { Cement, Portland } \ldots \ldots \ldots \ldots \ldots \ldots \ldots \ldots\end{array}$ & $\begin{array}{l}\text { set (3) } \\
\text { set } \\
40 \mathrm{~g} \\
\text { set } \\
\text { set } \\
\text { set }\end{array}$ & $\begin{array}{l}25.00 \\
35.00 \\
32.50 \\
10.00 \\
27.50 \\
27.50\end{array}$ \\
\hline $\begin{array}{r}748 \\
755 \\
756 \\
803 a \\
\text { D803a }\end{array}$ & 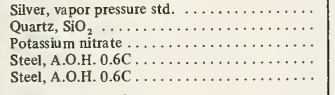 & $\begin{array}{l}\text { ea } \\
2 \mathrm{~g} \\
5 \mathrm{~g} \\
\text { ea } \\
\text { ea }\end{array}$ & $\begin{array}{l}75.00 \\
35.00 \\
35.00 \\
30.00 \\
35.00\end{array}$ & $\begin{array}{l}1013 \\
1014 \\
1015 \\
1016 \\
1019\end{array}$ & 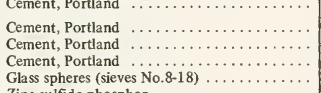 & $\begin{array}{l}\text { set } \\
\text { set } \\
\text { set } \\
100 \mathrm{~g}\end{array}$ & $\begin{array}{l}27.50 \\
27.50 \\
27.50 \\
27.50 \\
30.50\end{array}$ \\
\hline $\begin{array}{r}804 \mathrm{a} \\
805 \mathrm{a} \\
\mathrm{D} 805 \mathrm{a} \\
807 \mathrm{a} \\
\mathrm{D} 807 \mathrm{a}\end{array}$ & $\begin{array}{l}\text { Steel, basic electric } \ldots \ldots \ldots \ldots \ldots \ldots \ldots \ldots \\
\text { Steel, medium manganese } \ldots \ldots \ldots \ldots \ldots \ldots \\
\text { Steel, medium manganese } \ldots \ldots \ldots \ldots \ldots \ldots \\
\text { Steel, chromium-vanadium } \ldots \ldots \ldots \ldots \ldots \\
\text { Steel, chromium-vanadium } \ldots \ldots \ldots \ldots \ldots \ldots\end{array}$ & $\begin{array}{l}\text { ea } \\
\text { ea } \\
\text { ea } \\
\text { ea } \\
\text { ea }\end{array}$ & $\begin{array}{l}30.00 \\
30.00 \\
35.00 \\
30.00 \\
35.00\end{array}$ & $\begin{array}{l}1020 \\
1021 \\
1022 \\
1023\end{array}$ & $\begin{array}{l}\text { Zinc sulfide phosphor } \ldots \ldots \ldots \ldots \ldots \ldots \ldots \\
\text { Zinc silicate phosphor } \ldots \ldots \ldots \ldots \ldots \ldots \ldots \ldots \\
\text { Zinc sulfide phosphor } \ldots \ldots \ldots \ldots \ldots \ldots \ldots \\
\text { Zinc-cadmium sulfide phosphor } \\
\text { (Ag activator) } \ldots \ldots \ldots \ldots \ldots \ldots \ldots \ldots \ldots\end{array}$ & $\begin{array}{l}14 \mathrm{~g} \\
28 \mathrm{~g} \\
14 \mathrm{~g}\end{array}$ & $\begin{array}{l}23.50 \\
23.50 \\
23.50 \\
23.50\end{array}$ \\
\hline $\begin{array}{r}808 a \\
809 b\end{array}$ & $\begin{array}{l}\text { Steel, chromium-nickel } \ldots \ldots \ldots \ldots \ldots \ldots \ldots \ldots \\
\text { Steel, nickel } \ldots \ldots \ldots \ldots \ldots \ldots \ldots \ldots \ldots\end{array}$ & ea & $\begin{array}{l}30.00 \\
30.00\end{array}$ & 1024 & $\begin{array}{l}\text { Zinc-cadmium sulfide phosphor } \\
\text { (Cu activator) } \ldots \ldots \ldots \ldots \ldots \ldots \ldots \ldots \ldots \ldots\end{array}$ & $14 \mathrm{~g}$ & 23.50 \\
\hline $\begin{array}{r}\text { D809b } \\
810 \mathrm{a} \\
817 \mathrm{a}\end{array}$ & $\begin{array}{l}\text { Steel, nickel } \ldots \ldots \ldots \ldots \\
\text { Steel, Cr2-Mol } \ldots \ldots \ldots \ldots \ldots \\
\text { Steel, B.O.H. } 0.4 \mathrm{C} \ldots \ldots \ldots \ldots \ldots\end{array}$ & $\begin{array}{l}\text { ea } \\
\text { ea } \\
\text { ea }\end{array}$ & $\begin{array}{l}35.00 \\
30.00 \\
30.00\end{array}$ & $\begin{array}{l}1025 \\
1026 \\
1027\end{array}$ & $\begin{array}{l}\text { Zinc phosphate phosphor } \ldots \ldots \ldots \ldots \ldots \ldots \\
\text { Calcium tungstate phosphor } \ldots \ldots \ldots \ldots \ldots \\
\text { Magnesium tungstate phosphor } \ldots \ldots \ldots \ldots \ldots\end{array}$ & $\begin{array}{l}28 \mathrm{~g} \\
28 \mathrm{~g} \\
28 \mathrm{~g}\end{array}$ & $\begin{array}{l}23.50 \\
23.50 \\
23.50\end{array}$ \\
\hline $\begin{array}{r}820 \mathrm{a} \\
\text { D820a }\end{array}$ & 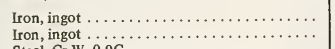 & ea & 30.00 & $\begin{array}{l}1028 \\
1029\end{array}$ & $\begin{array}{l}\text { Zinc silicate phosphor } \ldots \ldots \ldots \ldots \ldots \ldots \ldots \\
\text { Calcium silicate phosphor } \ldots \ldots \ldots \ldots \ldots \ldots\end{array}$ & $\begin{array}{l}28 \mathrm{~g} \\
14 \mathrm{~g}\end{array}$ & 23.50 \\
\hline $\begin{array}{l}821 \\
827 \\
836\end{array}$ & $\begin{array}{l}\text { Steel, Cr-W, } 0.9 \mathrm{C} \ldots \ldots \\
\text { Steel, Cr-Mo (boron only) (SAE } 4150) \ldots \ldots \\
\text { Steel, special (Cr6-Mo 3-W10) } \ldots \ldots \ldots \ldots\end{array}$ & ea & $\begin{array}{l}30.00 \\
30.00 \\
42.50\end{array}$ & $\begin{array}{l}1030 \\
1031 \\
1032\end{array}$ & $\begin{array}{l}\text { Magnesium arsenate phosphor } \ldots \ldots \ldots \ldots \ldots \\
\text { Calcium halophosphate phosphor } \ldots \ldots \ldots \ldots \\
\text { Barium silicate phosphor } \ldots \ldots \ldots \ldots \ldots\end{array}$ & $\begin{array}{l}28 \mathrm{~g} \\
28 \mathrm{~g} \\
28 \mathrm{~g}\end{array}$ & $\begin{array}{l}23.50 \\
23.50 \\
23.50\end{array}$ \\
\hline $\begin{array}{r}\text { D836 } \\
837\end{array}$ & $\begin{array}{l}\text { Steel, special (Cr6-Mo3-W10) } \ldots \ldots \ldots \ldots \ldots \\
\text { Steel, special (Cr8-Mo 2-W3-Co3) } \ldots \ldots \ldots \ldots\end{array}$ & $\begin{array}{l}\text { ea } \\
\text { ea }\end{array}$ & $\begin{array}{l}50.00 \\
42.50\end{array}$ & $\begin{array}{l}1033 \\
1051 b\end{array}$ & $\begin{array}{l}\text { Calcium phosphate phosphor } \ldots \ldots \ldots \ldots \ldots \\
\text { Barium cyclohexanebutyrate } \ldots \ldots \ldots \ldots \ldots\end{array}$ & $\begin{array}{r}28 \mathrm{~g} \\
5 \mathrm{~g}\end{array}$ & $\begin{array}{l}23.50 \\
31.00\end{array}$ \\
\hline $\begin{array}{r}\text { D837 } \\
838\end{array}$ & $\begin{array}{l}\text { Steel, special (Cr8-Mo2-W3-Co3) } \ldots \ldots \ldots \ldots \\
\text { Steel, Mo high speed (A1S1-SAE-M30) . . . . . }\end{array}$ & ea & $\begin{array}{l}50.00 \\
42.50\end{array}$ & $1052 b$ & $\begin{array}{l}\text { Bis(1-phenyl-1,3-butanediono) } \\
\text { oxovanadium (1V) } \ldots \ldots \ldots \ldots \ldots \ldots \ldots \ldots\end{array}$ & $5 \mathrm{~g}$ & 31.00 \\
\hline D838 & Steel, Mo high speed (A1SI-SAE-M 30) ....... & ea & 50.00 & $1053 \mathrm{a}$ & $\begin{array}{l}\text { Cadmium cyclohexanebutyrate } \ldots \ldots \ldots \ldots \ldots \\
\text { Cobalt cyclohexanebutyrate } \ldots \ldots \ldots \ldots \ldots\end{array}$ & $5 \mathrm{~g}$ & $\begin{array}{l}31.00 \\
31.00\end{array}$ \\
\hline $\begin{array}{r}839 \\
\text { D839 } \\
840\end{array}$ & $\begin{array}{l}\text { Steel, Mo high speed (A1Sl-SAE-M 36) } \ldots \ldots \ldots \\
\text { Steel, Mo high speed (AISl-SAE-M } 36 \text { ) . . . . } \\
\text { Steel, special W high speed }\end{array}$ & $\begin{array}{l}\text { ea } \\
\text { ea }\end{array}$ & $\begin{array}{l}42.50 \\
50.00\end{array}$ & $\begin{array}{l}1055 \mathrm{~b} \\
1057 \mathrm{~b} \\
1059 \mathrm{~b}\end{array}$ & $\begin{array}{l}\text { Cobalt cyclohexanebutyrate } \ldots \ldots \ldots \ldots \ldots \\
\text { Dibutyltin bis(2-ethylhexanoate) } \ldots \ldots \ldots \ldots \\
\text { Lead cyclohexanebutyrate } \ldots \ldots \ldots \ldots \ldots\end{array}$ & $\begin{array}{l}5 \mathrm{~g} \\
5 \mathrm{~g} \\
5 \mathrm{~g}\end{array}$ & $\begin{array}{l}31.00 \\
31.00 \\
31.00\end{array}$ \\
\hline $\begin{array}{r}\text { D840 } \\
841\end{array}$ & $\begin{array}{l}\text { (Cr2-W13-Col 2) } \ldots \ldots \ldots \ldots \ldots \ldots \ldots \ldots \\
\text { Steel, special W high speed } \\
\text { (Cr2-W13-Co1 2) } \ldots \ldots \ldots \ldots \ldots \ldots \ldots \ldots \\
\text { Steel, W high speed (AISI-SAE-TI) } \ldots \ldots \ldots \ldots\end{array}$ & $\begin{array}{l}\text { ea } \\
\text { ea } \\
\text { ea }\end{array}$ & $\begin{array}{l}42.50 \\
50.00 \\
42.50\end{array}$ & $\begin{array}{l}1060 \mathrm{a} \\
1061 \mathrm{c} \\
1062 \mathrm{a}\end{array}$ & $\begin{array}{l}\text { Lithium cyclohexanebutyrate } \ldots \ldots \ldots \ldots \\
\text { Magnesium cyclohexanebutyrate } \ldots \ldots \ldots \ldots \\
\text { Manganous cyclohexanebutyrate . . . . . }\end{array}$ & $\begin{array}{l}5 \mathrm{~g} \\
5 \mathrm{~g} \\
5 \mathrm{~g}\end{array}$ & $\begin{array}{l}31.00 \\
31.00 \\
31.00\end{array}$ \\
\hline
\end{tabular}




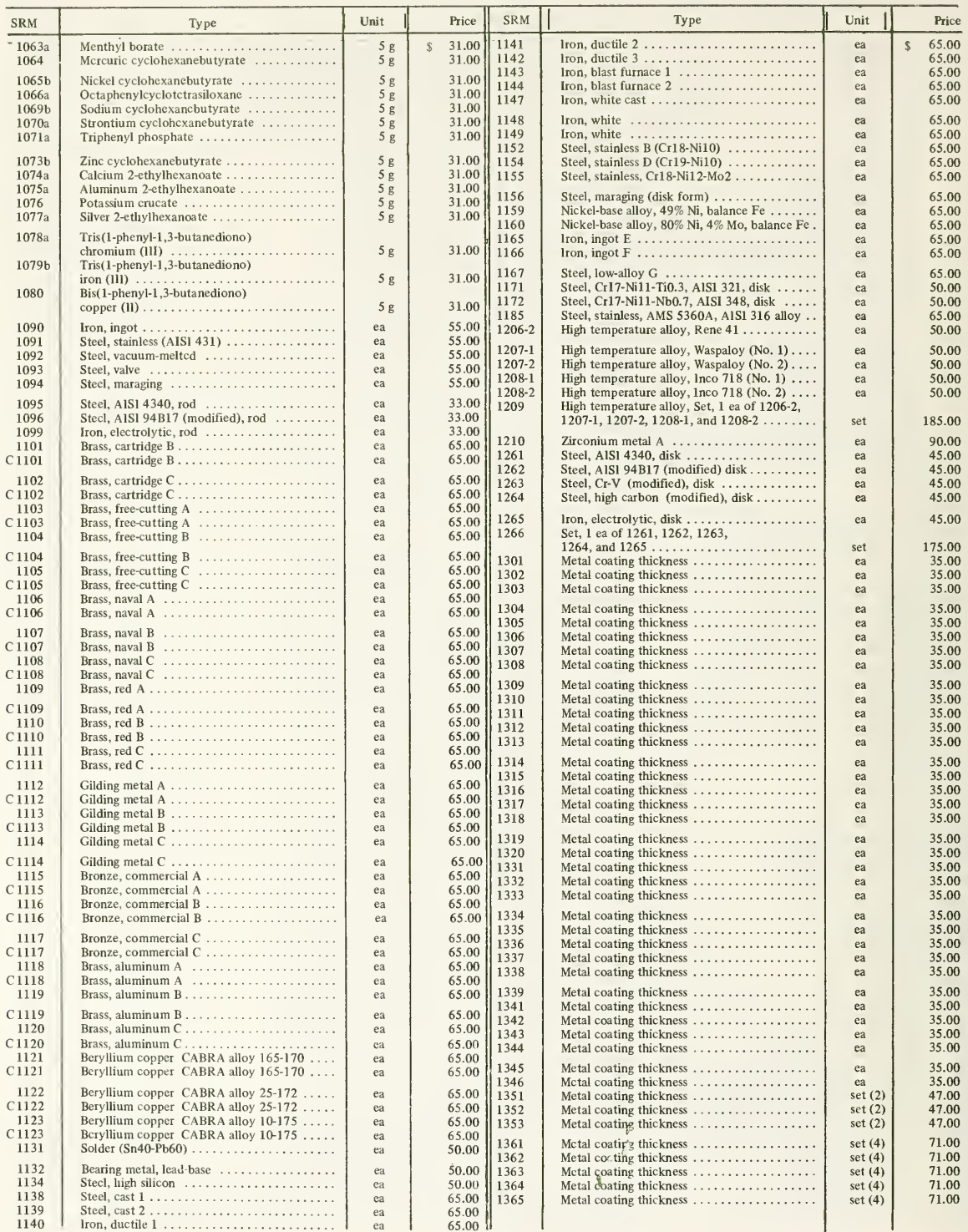




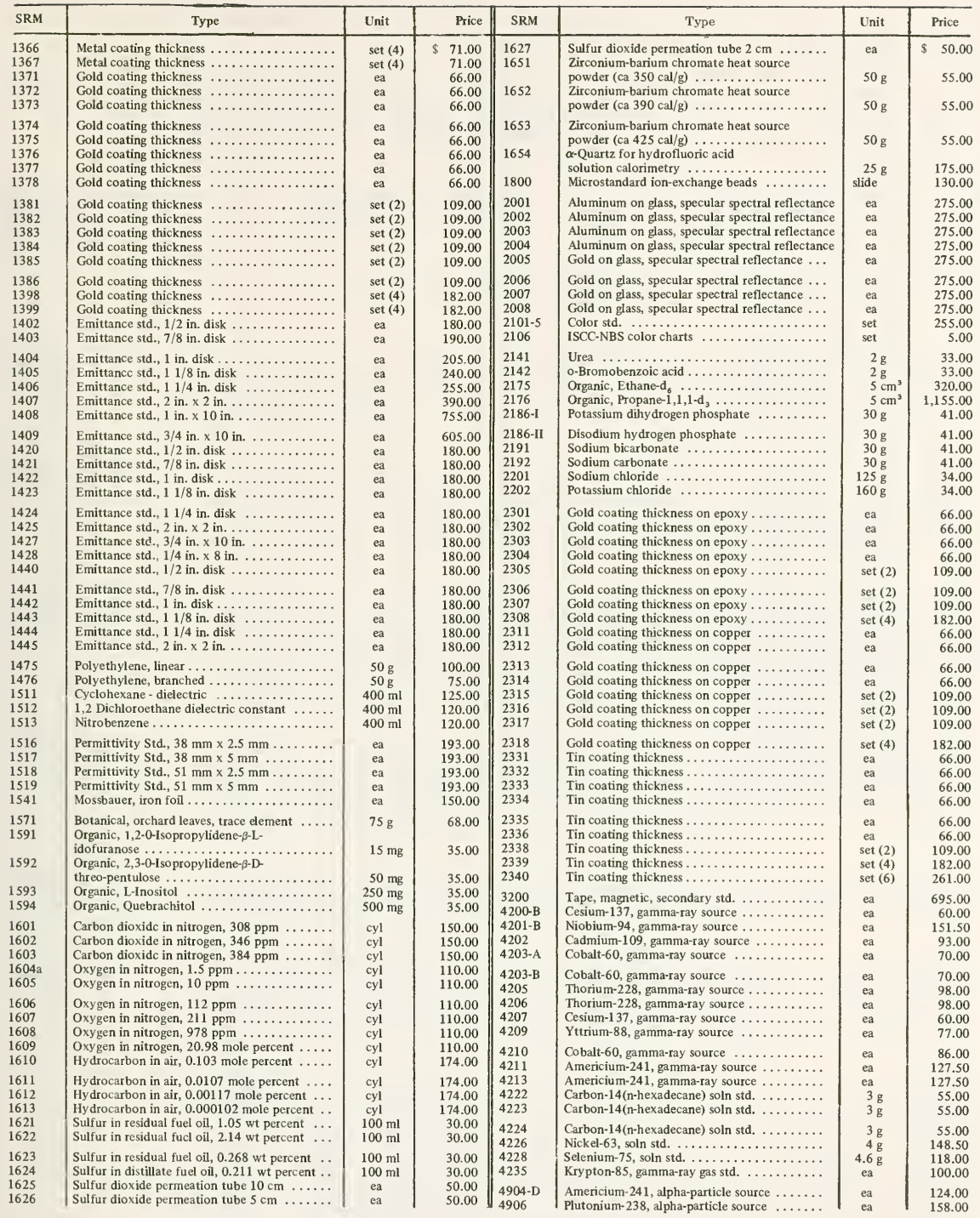




\begin{tabular}{|c|c|c|c|c|c|c|c|}
\hline SRM & Type & Unit & Price & & B. RESEARCH MATERIALS & & \\
\hline $\begin{array}{l}4921-C \\
4922-E \\
4925\end{array}$ & $\begin{array}{l}\text { Sodium- } 22, \text { soln std. } \ldots \ldots \ldots \ldots \ldots \ldots \ldots \ldots \ldots \\
\text { Sodium- } 22 \text {, soln std. } \ldots \ldots \ldots \ldots \ldots \ldots \ldots \ldots \\
\text { Carbon-14 (benzoic acid in tolucne) } \ldots \ldots \ldots \ldots\end{array}$ & $\begin{array}{l}3 \mathrm{~g} \\
5 \mathrm{~g} \\
3 \mathrm{~g}\end{array}$ & $\begin{array}{l}42.00 \\
61.00 \\
48.00\end{array}$ & RM & Type & Unit & Price \\
\hline $\begin{array}{l}4926 \\
4927\end{array}$ & $\begin{array}{l}\text { Hydrogen }-3 \text { (watcr) } \ldots \ldots \ldots \ldots \ldots \ldots \ldots \ldots \ldots \ldots \ldots \ldots \ldots \ldots \\
\text { Hydrogen }-3 \text { (watcr) } \ldots \ldots \ldots \ldots \ldots \ldots\end{array}$ & $\begin{array}{r}25 \mathrm{~g} \\
3 \mathrm{~g}\end{array}$ & 48.00 & $\begin{array}{l}\text { RM-1C } \\
\text { RM-1R }\end{array}$ & $\begin{array}{l}\text { Ultra-purity aluminum, single crystal cubc } \ldots . \\
\text { Ultra-purity aluminum, polycrystaline rod } \ldots .\end{array}$ & $\begin{array}{l}\text { ea } \\
\text { ea }\end{array}$ & $\begin{array}{r}\$ 90.00 \\
50.00\end{array}$ \\
\hline $\begin{array}{l}4929-C \\
4935-C \\
4940-B\end{array}$ & 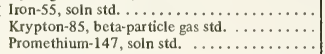 & $\begin{array}{c}4 \mathrm{~g} \\
10 \mathrm{ml} \\
3 \mathrm{~g}\end{array}$ & $\begin{array}{r}115.00 \\
100.00 \\
60.00\end{array}$ & \multicolumn{4}{|c|}{ C. GENERAL MATERIALS } \\
\hline $\begin{array}{l}4941-\mathrm{C} \\
4943\end{array}$ & $\begin{array}{l}\text { Cobalt- } 57, \text { soln std. } \ldots \ldots \ldots \ldots \ldots \ldots \ldots \ldots \ldots \ldots \ldots \ldots \ldots \ldots \ldots \ldots \\
\text { Chlorine- } 36, \text { soln std. } \ldots \ldots \ldots \ldots \ldots\end{array}$ & $\begin{array}{l}5 \mathrm{~g} \\
3 \mathrm{~g}\end{array}$ & $\begin{array}{r}108.00 \\
43.00\end{array}$ & GM & Type & Unit & Price \\
\hline $\begin{array}{l}4947 \\
4948 \\
4950-B\end{array}$ & $\begin{array}{l}\text { Hydrogen-3 (tritiated toluene) } \ldots \ldots \ldots \ldots \ldots \\
\text { Cerium-Praseodymium-144, soln std. . . . . . } \\
\text { Radium solution std., } 10^{-9} \mathrm{~g} \text { (Rd analysis) } \ldots \ldots\end{array}$ & $\begin{array}{r}4 \mathrm{~g} \\
3.3 \mathrm{~g} \\
20 \mathrm{~g}\end{array}$ & $\begin{array}{l}46.00 \\
70.00 \\
81.00\end{array}$ & GM-1 & $\begin{array}{l}\text { Hydrogen in steel } \\
\text { Hydrogen in steel }\end{array}$ & $\begin{array}{r}\text { set } \\
\text { set }\end{array}$ & $\begin{array}{r}\$ 86.00 \\
86.00\end{array}$ \\
\hline $\begin{array}{l}4951 \\
4952-\mathrm{A} \\
4953 \\
4955 \\
4956\end{array}$ & $\begin{array}{l}\text { Radium solution std., } 10^{-11} \mathrm{~g} \text { (Rd analysis) } \ldots \\
\text { Radium blank solution (Rd analysis) } \ldots \ldots \ldots . \\
\text { Radium, } 10^{-8} \mathrm{~g} \text { (Rd analysis) } \ldots \ldots \ldots \ldots \ldots \\
\text { Radium solution std., } 0.1 \mu \mathrm{g} \mathrm{Ra} \ldots \ldots \ldots \ldots \\
\text { Radium solution std., } 0.2 \mu \mathrm{g} \mathrm{Ra} \ldots \ldots \ldots \ldots\end{array}$ & $\begin{array}{r}100 \mathrm{~g} \\
100 \mathrm{~g} \\
20 \mathrm{~g} \\
5 \mathrm{~g} \\
5 \mathrm{~g}\end{array}$ & $\begin{array}{l}48.00 \\
30.00 \\
81.00 \\
63.00 \\
63.00\end{array}$ & & & & \\
\hline $\begin{array}{l}4957 \\
4958 \\
4959 \\
4960\end{array}$ & $\begin{array}{l}\text { Radium solution std., } 0.5 \mu \mathrm{g} \mathrm{Ra} \ldots \ldots \ldots \ldots \\
\text { Radium solution std., } 1 \mu \mathrm{g} \mathrm{Ra} \ldots \ldots \ldots \ldots \ldots \\
\text { Radium solution std., } 2 \mu \mathrm{g} \mathrm{Ra} \ldots \ldots \ldots \ldots \ldots \\
\text { Radium solution std., } 5 \mu \mathrm{g} \mathrm{Ra} \ldots \ldots \ldots \ldots\end{array}$ & $\begin{array}{l}5 \mathrm{~g} \\
5 \mathrm{~g} \\
5 \mathrm{~g} \\
5 \mathrm{~g}\end{array}$ & $\begin{array}{l}63.00 \\
63.00 \\
63.00 \\
63.00\end{array}$ & & & & \\
\hline $\begin{array}{l}4961 \\
4962 \\
4963 \\
4964-B\end{array}$ & $\begin{array}{l}\text { Radium solution std., } 10 \mu \mathrm{g} \mathrm{Ra} \ldots \ldots \ldots \ldots \ldots \\
\text { Radium solution std., } 20 \mu \mathrm{g} \mathrm{Ra} \ldots \ldots \ldots \ldots \ldots \\
\text { Radium solution std., } 50 \mu \mathrm{g} \text { Ra........... } \\
\text { Radium solution std., } 102 \mu \mathrm{g} \text { Ra......... }\end{array}$ & $\begin{array}{l}5 \mathrm{~g} \\
5 \mathrm{~g} \\
5 \mathrm{~g} \\
5 \mathrm{~g}\end{array}$ & $\begin{array}{l}63.00 \\
63.00 \\
63.00 \\
63.00\end{array}$ & & & & \\
\hline $\begin{array}{l}4990-B \\
4991-C \\
4996-B \\
4998-E\end{array}$ & $\begin{array}{l}\text { Carbon-14, contemporary std. for dating } \ldots \ldots \\
\text { Sodium- } 22 \text {, gamma-ray source } \ldots \ldots \ldots \ldots \ldots \ldots \\
\text { Sodium- } 22 \text {, gamma-ray source } \ldots \ldots \ldots \ldots \ldots \\
\text { Yttrium- } 88 \text {, gamma-ray source } \ldots \ldots \ldots \ldots \ldots\end{array}$ & $\begin{array}{l}1 \mathrm{lb} \\
\mathrm{ea} \\
\mathrm{ea} \\
\mathrm{ea}\end{array}$ & $\begin{array}{l}26.50 \\
79.00 \\
79.00 \\
77.00\end{array}$ & & & & \\
\hline $\begin{array}{l}\text { U-0002 } \\
\text { U-005 } \\
\text { U-010 } \\
\text { U-015 } \\
\text { U-020 }\end{array}$ & $\begin{array}{l}\text { Uranium oxide - depleted (U-235) } \ldots \ldots \ldots \ldots \\
\text { Uranium oxide - depleted (U-235) } \ldots \ldots \ldots \ldots \\
\text { Uranium oxide - enriched (U-235) } \ldots \ldots \ldots \ldots \\
\text { Uranium oxide - enriched (U-235) } \ldots \ldots \ldots \ldots \\
\text { Uranium oxide - enriched (U-235) } \ldots \ldots \ldots \ldots\end{array}$ & $\begin{array}{l}1 \mathrm{~g} \\
1 \mathrm{~g} \\
1 \mathrm{~g} \\
1 \mathrm{~g} \\
1 \mathrm{~g}\end{array}$ & $\begin{array}{l}58.50 \\
48.50 \\
48.50 \\
48.50 \\
49.00\end{array}$ & & & & \\
\hline $\begin{array}{l}\text { U-030 } \\
\text { U-050 } \\
\text { U-100 } \\
\text { U-150 } \\
\text { U-200 }\end{array}$ & 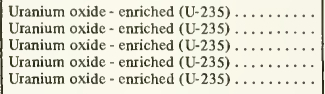 & $\begin{array}{l}1 \mathrm{~g} \\
1 \mathrm{~g} \\
1 \mathrm{~g} \\
1 \mathrm{~g} \\
1 \mathrm{~g}\end{array}$ & $\begin{array}{l}49.00 \\
49.00 \\
50.00 \\
51.00 \\
51.50\end{array}$ & & & & \\
\hline $\begin{array}{l}\text { U-350 } \\
\text { U-500 } \\
\text { U-750 } \\
\text { U-800 } \\
\text { U-850 }\end{array}$ & $\begin{array}{l}\text { Uranium oxide - enriched }(U-235) \ldots \ldots \ldots \ldots \\
\text { Uranium oxide - enriched (U-235) } \ldots \ldots \ldots \ldots \\
\text { Uranium oxide - enriched (U-235) } \ldots \ldots \ldots \ldots \\
\text { Uranium oxide - enriched (U-235) } \ldots \ldots \ldots \ldots \\
\text { Uranium oxide - enriched (U-235) } \ldots \ldots \ldots \ldots\end{array}$ & $\begin{array}{l}1 \mathrm{~g} \\
1 \mathrm{~g} \\
1 \mathrm{~g} \\
1 \mathrm{~g} \\
1 \mathrm{~g}\end{array}$ & $\begin{array}{l}54.50 \\
56.00 \\
61.50 \\
62.00 \\
63.00\end{array}$ & & & & \\
\hline $\begin{array}{l}\text { U-900 } \\
\text { U-930 } \\
\text { U-970 }\end{array}$ & $\begin{array}{l}\text { Uranium oxide - enriched (U-235) } \ldots \ldots \ldots \ldots \\
\text { Uranium oxide - enriched (U-235) } \ldots \ldots \ldots \ldots \\
\text { Uranium oxide - enriched (U-235) } \ldots \ldots \ldots \ldots\end{array}$ & $\begin{array}{l}1 \mathrm{~g} \\
1 \mathrm{~g} \\
1 \mathrm{~g}\end{array}$ & $\begin{array}{l}64.00 \\
65.50 \\
68.50\end{array}$ & & & & \\
\hline
\end{tabular}




\section{STANDARD REFERENCE MATERIALS NEW - RENEWALS}

\section{Category 3.1. Steels (Chip Form)}

SRM $\quad 20 \mathrm{~g}$

SRM 121d

SRM $123 c$

SRM $125 b$

SRM $166 \mathrm{c}$

SRM 361 366

Steel, AISI 1045 in chip form has been issued with a Certificate of Analysis. The composition is: C 0.462 , Mn 0.665, P 0.012, S 0.028, Si 0.305, Cu 0.034, Ni 0.034, Cr 0.036, V 0.002, Mo 0.008, and Al 0.040. This material costs $\$ 33.00$ per $150 \mathrm{~g}$ unit.

Steel, stainless, Cr17-Ni11-Ti0.3, AISI 321, in chip form has been issued with a Certificate of Analysis. The nominal composition is: C $0.07, \mathrm{Mn} \mathrm{1.8,} \mathrm{P} \mathrm{0.02,} \mathrm{S}$ 0.01, Si 0.5, Cu 0.1, Ni 11.2, Cr 17.4, Mo 0.2, Ti 0.3, and Co 0.1. This material is also available in disk form as SRM 1171, see Category 3.2. SRM 121d costs $\$ 33.00$ per $150 \mathrm{~g}$ unit.

Steel, stainless, Cr17-Ni1 1-Nb0.7, AISI 348, in chip form has been issued with a Certificate of Analysis. The nominal composition is: $\mathrm{C} 0.05, \mathrm{Mn} \mathrm{1.7,} \mathrm{P} \mathrm{0.01,}$ S 0.01, Si 0.6, Cu 0.1, Ni 11.4, Cr 17.4, V 0.03, Mo 0.2, Nb 0.7, Ta 0.001, and Co 0.1. This material is also available in disk form as SRM 1172, see Category 3.2. SRM $123 \mathrm{c}$ costs $\$ 33.00$ per $150 \mathrm{~g}$ unit.

High Silicon Steel in chip form has been issued with a Certificate of Analysis. The nominal composition is: C 0.028, Mn 0.278, P 0.029, S 0.008, Si 2.89, Cu 0.071 , Ni 0.038, Cr 0.019, Mo 0.008, Sn 0.003 and Al 0.329. This material is priced at $\$ 33.00$ per $150 \mathrm{~g}$ unit. A high silicon steel of similar composition is also issued in solid disk form as SRM 1134 in Category 3.2.

Low Carbon Stainless Steel (AISI 316L) in powder form has been issued with a Certificate of Analysis for carbon. The nominal value is $0.0078 \%$. The material is available in 100 gram units priced at $\$ 25.00$.

Low alloy steel and electrolytic iron standards in chip form for chemical analysis--companion SRM's to the "1200 series" (See Category 3.2)-have been issued with Provisional Certificates of Analysis. These SRM's are sold as follows:

\begin{tabular}{l|l|c|r} 
SRM & \multicolumn{1}{|c|}{ Type } & Unit & Price \\
\hline 361 & Steel, AISI 4340 & $150 \mathrm{~g}$ & $\$ 33.00$ \\
362 & Steel, AISI 94B17 (modified) & $150 \mathrm{~g}$ & 33.00 \\
363 & Steel, Cr-V (modified) & $150 \mathrm{~g}$ & 33.00 \\
364 & Steel, High Carbon (modified) & $150 \mathrm{~g}$ & 33.00 \\
365 & Iron, Electrolytic & $150 \mathrm{~g}$ & 33.00 \\
366 & Set of one each 361, 362, 363, 364, and 365 & set & 100.00
\end{tabular}


SRM $661-$ 668

SRM 1095

SRM 1096

SRM 1099

SRM 1134

SRM 1171

SRM 1172

Low alloy steel and electrolytic iron standards in solid form for microchemical methods of analysis such as electron probe, laser probe, and spark source mass spectrometry--from the same melts as the "1200 Series" (See below)--have been issued with Provisional Certificates of Analysis. These SRM's are issued in sets consisting of either two or five rods, which are $3.2 \mathrm{~mm}$ in diameter and $51 \mathrm{~mm}$ long. SRM 664 is available as a single SRM.

\begin{tabular}{l|l|c|r} 
SRM & \multicolumn{1}{|c|}{ Type } & Unit & Price \\
\hline 661 & Steel, AISI 4340 & & \\
662 & Steel, AISI 94B17 (modified) & & \\
663 & Steel, Cr-V (modified) & ea & $\$ 25.00$ \\
664 & Steel, High Carbon (modified) & & \\
665 & Iron, Electrolytic & sets & 40.00 \\
666 & Set of 2 rods: 661 and 665 & sets & 40.00 \\
667 & Set of 2 rods: 662 and 663 & sets & 75.00
\end{tabular}

Steel, AISI 4340 in solid form for determination of oxygen in metal by vaccuum or inert gas fusion and neutron activation methods of analysis-from the same melt as 1261 (See below)-has been issued with a Certificate of Analysis for oxygen at $9 \mathrm{ppm}$. This SRM is a rod $6.4 \mathrm{~mm}$ in diameter and $102 \mathrm{~mm}$ long, and costs $\$ 33.00$ per unit.

Steel, AISI 94B17 (modified) in solid form for the determination of oxygen and nitrogen in metal by vacuum or inert gas fusion and neutron activation methods of analysis--from the same melt as 1262 (see below)-has been issued with a Certificate of Analysis. The values for oxygen and nitrogen are $10 \mathrm{ppm}$ and $40 \mathrm{ppm}$, respectively. This SRM is a rod $6.4 \mathrm{~mm}$ in diameter and $102 \mathrm{~mm}$ long, and costs $\$ 33.00$ each.

Electrolytic Iron in solid form for the determination of oxygen in metals by vacuum or inert gas fusion and neutron activation methods of analysis-from the same melt as 1265 (See below)-has been issued with a Provisional Certificate of Analysis for oxygen at $61 \mathrm{ppm}$. This SRM is a rod $6.4 \mathrm{~mm}$ in diameter and $102 \mathrm{~mm}$ long, and costs $\$ 33.00$ per unit.

High Silicon Steel in solid form has been issued with a Certificate of Analysis. The nominal composition is: C $0.026, \mathrm{Mn} \mathrm{0.277}, \mathrm{P} 0.028, \mathrm{~S} 0.009, \mathrm{Si} 2.89, \mathrm{Cu}$ 0.070 , Ni 0.038 , Cr 0.019 , Mo 0.008 , Sn 0.003 and $\mathrm{Al} 0.329$. This material is issued in the form of a disc $11 / 4$ inch $(31.8 \mathrm{~mm})$ in diameter and $3 / 4 \mathrm{inch}$ $(19.1 \mathrm{~mm})$ thick, priced at $\$ 50.00$ each. A similar material is available in chip form as SRM 125b in Category 3.1 .

Steel, stainless, Cr17-Ni11-Ti0.3, AISI 321, in disk form, $31 \mathrm{~mm}$ in diameter and $19 \mathrm{~mm}$ thick, has been issued with a Certificate of Analysis. The nominal composition is: $\mathrm{C} 0.07, \mathrm{Mn} 1.8, \mathrm{P} 0.02$, $\mathrm{Si} 0.5, \mathrm{Cu} 0.1$, Ni 11.2, $\mathrm{Cr} 17.4$, Mo 0.2 , Ti 0.3 , and Co 0.1. This material is also available in chip form as SRM $121 \mathrm{~d}$, see Category 3.1. SRM 1171 costs $\$ 50.00$ ea.

Steel, stainless, Cr17-Ni11-Nb0.7, AISI 348, in disk form, $31 \mathrm{~mm}$ in diameter and $19 \mathrm{~mm}$ thick, has been issued with a Certificate of Analysis. The nominal composition is: C $0.05, \mathrm{Mn} \mathrm{1.7,} \mathrm{P} 0.01, \mathrm{~S} 0.01$, Si $0.6, \mathrm{Cu} 0.1$, Ni 11.4, Cr $17.4, \mathrm{~V} 0.03$, Mo $0.2, \mathrm{Nb} 0.7$, Ta 0.001 , and $\mathrm{Co} 0.1$. This material is also available in chip form as SRM $123 \mathrm{c}$. SRM 1172 costs $\$ 50.00$ ea. 
SRM $1206-$

1209

SRM $1261-$ 1266

SRM 53e

SRM $654 \mathrm{a}$

SRM 1132

\section{SRM 483}

Five SRM's for three important high-temperature alloys have been made available with a Provisional Certificate of Analysis (obtainable on request). One is for high-temperature alloy Rene 41 (1206-2), while two each are for the hightemperature alloys Waspaloy (1207-1 and 1207-2) and Inco 718 (1208-1 and 1208-2). Issued in the form of solid sections, approximately $31 \mathrm{~mm}$ square and $19 \mathrm{~mm}$ thick, the standards are designed primarily for application in $\mathrm{x}$-ray spectrometric methods of analysis. However, they also may be used in optical emission spectrometric methods of analysis. These SRM's cost $\$ 50.00$ per unit, or may be purchased as a complete set (as SRM 1209) for $\$ 185.00$ per set.

Low alloy steel and electrolytic iron-the "1200 Series" (replacements for the 1100 series)--have been issued with Provisional Certificates of Analysis (obtainable on request) for use in optical emission and x-ray spectrometric analysis. These SRM's are disks $31 \mathrm{~mm}$ in diameter and $19 \mathrm{~mm}$ thick. The initial certification is made for some 10 to 15 elements; however, chemical information is provided for the remaining 40 elements. They are sold as follows:

\begin{tabular}{l|l|c|c} 
SRM & \multicolumn{1}{|c|}{ Type } & Unit & Price \\
\hline 1261 & Steel, AISI 4340 & ea & $\$ 45.00$ \\
1262 & Steel, AISI 94B17 (modified) & ea & 45.00 \\
1263 & Steel, Cr-V (modified) & ea & 45.00 \\
1264 & Steel, High Carbon (modified) & ea & 45.00 \\
1265 & Iron, Electrolytic & ea & 45.00 \\
1266 & Set of one each 1261, 1262, 1263, 1264, and 1265 & set & 175.00
\end{tabular}

Category 3.6. Nonferrous Alloys (Chip Form)

Lead base bearing metal in powder form has been issued with a Certificate of Analysis. The nominal composition is: ( $\mathrm{Pb} 84$, not certified), $\mathrm{Sb} 10.26$, Sn 5.84, $\mathrm{Cu} 0.054, \mathrm{Bi} 0.052$, As 0.057 and $\mathrm{Ni} 0.003$. This material is the same as SRM 1132 which is issued in the solid form in Category 3.7. and is priced at $\$ 33.00$ per $150 \mathrm{~g}$ unit.

\section{Category 3.7. Nonferrous Alloys (Solid Form)}

Titanium Alloy, $6 \mathrm{Al}-4 \mathrm{~V}$ has been issued with a Certificate of Analysis. The material is in the form of a disk $31 \mathrm{~mm}(11 / 4 \mathrm{in})$ in diameter and $6.4 \mathrm{~mm}$ (1/4 in) thick with a nominal composition of: Al 6.3 and V 3.9 (values for Fe, $\mathrm{Cr}, \mathrm{Mn}$, and Mo are not certified, but are given for information only). This material costs $\$ 35.00$ each.

Lead base bearing metal in solid form has been issued with a Certificate of Analysis. The material is in the form of a disc $11 / 4$ inch $(31.8 \mathrm{~mm})$ in diameter and $3 / 4$ inch $(19.0 \mathrm{~mm})$ thick with a nominal composition of $(\mathrm{Pb} 84$, not certified), $\mathrm{Sb} 10.26$, Sn 5.84, Cu 0.054, Bi 0.052, As 0.057 and Ni 0.003. This material is the same as SRM $53 \mathrm{e}$ which is issued in a powder form and is listed in Category 3.6. SRM 1132 is priced at $\$ 50.00$ each.

\section{Category 3.8. Miscellaneous Metals}

Iron--3\% Silicon Alloy Microprobe Standard has been issued with a Certificate of Analysis. The material is $3 \mathrm{~mm}$ by $3 \mathrm{~mm}$ by $0.28 \mathrm{~mm}$ with a nominal composition of: Silicon $3.2 \mathrm{wt}$. percent and Iron (by difference) $96.8 \mathrm{wt}$. percent. This material costs $\$ 50.00$ each. 
Potassium Dichromate has been issued with a Provisional Certificate of Analysis. This is a primary chemical standard certified for purity based on effective oxidizing power, nominally $99.98 \%$. It is sold in 60 gram units priced at $\$ 26.00$.

SRM 723

tris (Hydroxymethyl)aminomethane-2-amino-2-hydroxymethyl-1, 3-propanediol--is the first basimetric SRM issued by NBS. The basimetric value certified is $99.9690 \pm 0.0030$ weight percent. The uncertainty represents the 95 percent confidence interval of the mean for 30 determinations. The corresponding standard deviation of a single measurement is 0.0081 units. SRM 723 costs $\$ 50.75$ per $50 \mathrm{~g}$ unit.

\section{Category 3.42. Microanalysis Standards}

SRM 2141

Urea is a compound with a relatively high nitrogen content, 46.65 percent, issued to supplement the other micronitrogen SRM's-acetanilide (SRM 141b), which contains an open-chain nitrogen atom, and nicotinic acid (SRM 148), which contains a heterocyclic nitrogen atom. Both $141 \mathrm{~b}$ and 148 have relatively low nitrogen contents of 10.36 and 11.38 percent, respectively. SRM 2141 costs $\$ 33.00$ per $2 \mathrm{~g}$ unit.

SRM 2142 o-Bromobenzoic acid is certified only for the weight percentage of bromine, but has been characterized for identity and purity by several organic and physical chemistry techniques. SRM 2142 is the first in a planned series of SRM's certified for halogens that are to be issued to augment the existing microchemical SRM's. SRM 2142 costs $\$ 33.00$ per $2 \mathrm{~g}$ unit.

\section{Category 3.43. Clinical Laboratory Standards}

SRM 916

Bilirubin has been issued with a Provisional Certificate of Analysis as a chemical of known purity for use as an analytical standard in clinical chemistry. The provisionally certified purity for bilirubin is 99.0 percent. This material costs $\$ 92.00$ per 100 mg unit.

SRM $917 D$-glucose is certified for use as an analytical standard in clinical chemistry. The certified purity is $99.9 \pm 0.1$ percent and the relative amounts of $\alpha$ - and $\beta$ - $D$ glucopyranose are given. SRM 917 costs $\$ 43.00$ per $25 \mathrm{~g}$ unit.

SRM 918

Potassium Chloride has been issued with a Certificate of Analysis as a chemical of known purity for use as an analytical standard for clinical chemistry. The certified purity is 99.9 percent. This material costs $\$ 40.00$ per $30 \mathrm{~g}$ unit.

SRM $922-$

923

SRM 930

Tris(hydroxymethyl)aminomethane and Tris(hydroxymethyl)aminomethane hydrochloride have been issued with a Provisional Certificate of Analysis for use as a $\mathrm{pH}$ standard for clinical chemistry. The Certificate provides directions for preparing a solution of known $\mathrm{pH}$ vaiue from the two SRM's, and provides a range of $\mathrm{pH}$ values as a function of solution temperature. SRM 922 costs $\$ 40.00$ per $125 \mathrm{~g}$ unit; SRM 923 costs $\$ 40.00$ per $160 \mathrm{~g}$ unit.

Glass Filters for Spectrophotometers have been issued with a Certificate. This SRM consists of three glass filters having transmittances of approximately 10 , 20 , and 30 percent. Each filter is individually calibrated and certified for absorbance and transmittance over a spectral wavelength range from 440 to 635 nanometers. These filters are intended to check the accuracy of the photometric scale of spectrophotometers, and to provide a means of interlaboratory 
comparisons of spectrophotometric data. It is probable that in the field of clinical chemistry a large amount of data are being obtained on precise instruments whose accuracy is unknown. To make these data more meaningful and universally applicable, the biases between instruments must be eliminated or at least determined. A major purpose of these filters will be to assure that systematic errors due to a particular characteristic or condition of an instrument can be recognized. This SRM costs $\$ 300.00$ per set of three filters.

\section{Category 3.44. Metallo-Organic Compounds}

SRM 1061c Magnesium cyclohexanebutyrate has been issued with a Provisional Certificate of Analysis. This SRM has a composition of 6.45 percent magnesium and costs $\$ 31.00$ per 5 g unit.

SRM 1079b Tris(1-phenyl-1,3-butanediono)iron (III) has been issued with a Certificate of Analysis. It has a nominal composition of $10.45 \%$ iron and is priced at $\$ 31.00$ per 5 gram unit.

\section{Category 3.46. Botanical Standards}

SRM 1571

Orchard Leaves has been issued with a Provisional Certificate of Analysis. This SRM is the first of a series of botanical standards to be certified for chemical elements. This material is certified for the following elements: $\mathrm{Ca}, \mathrm{K}, \mathrm{Fe}, \mathrm{Na}$, $\mathrm{Cu}$ and $\mathrm{Ni}$. The content of the following elements is given for information only: $\mathrm{Hg}, \mathrm{Pb}, \mathrm{N}, \mathrm{Mg}, \mathrm{P}, \mathrm{As}, \mathrm{Bi}, \mathrm{B}, \mathrm{Cr}, \mathrm{Co}, \mathrm{F}, \mathrm{Mn}, \mathrm{Se}, \mathrm{U}$, and $\mathrm{Zn}$. This SRM costs $\$ 68.00$ per $75 \mathrm{~g}$ unit.

\section{Category 3.51. Analyzed Gases}

SRM 1604a Oxygen in Nitrogen has been issued with a Certificate of Analysis. The nominal concentration of oxygen in nitrogen is $1.5 \mathrm{ppm}$. This SRM is sold in cylinders containing 68 liters at STP for \$1 10.00 per cylinder.

SRM 1610 - Certified Gas Standards (Hydrocarbon in Air) have been issued with a Certifi1613 cate of Analysis. The nominal hydrocarbon concentration calculated as methane is
1610
0.103 mole percent
1612
1611
.0107 mole percent
1613
.00117 mole percent .000102 mole percent

These SRM's are sold in cylinders containing 68 liters at STP, for \$174.00 per cylinder.

SRM 1625

1626

1627

Sulfur Dioxide Permeation Tubes are intended for calibrating air pollution monitoring apparatus, and may be used also for the verification of air pollution analytical methods and procedures. SRM's 1625, 1626, and 1627 have effective lengths of 10,5 , and $2 \mathrm{~cm}$, respectively. The permeation rate per $\mathrm{cm}$ of length is approximately $0.28 \mu \mathrm{g}$ of $\mathrm{SiO}_{2}$ per minute at $25^{\circ} \mathrm{C}$. Each tube is individually calibrated and its permeation rate is certified to one percent (relative) over the temperature range of 20 to $30^{\circ} \mathrm{C}$. These SRM's cost $\$ 50.00$ per unit.

\section{Category 3.52. Analyzed Liquids}

SRM 1623

Sulfur in Residual Fuel Oil has been issued with a Provisional Certificate of Analysis. The certified value for the sulfur content is $0.268 \mathrm{wt}$. percent. This material costs $\$ 30.00$ per $100 \mathrm{ml}$ unit.

SRM 1624 Sulfur in Distillate Fuel Oil has been issued with a Provisional Certificate of Analysis. The certified value for the sulfur content is $0.211 \mathrm{wt}$. percent. This material costs $\$ 30.00$ per $100 \mathrm{ml}$ unit. 


\section{Category 3.54. Ores}

SRM 180

High Grade Fluorspar has been issued with a Certificate of Analysis. The certified value of $\mathrm{CaF}_{2}$ is $98.8 \mathrm{wt}$. percent. This material has been issued for use by the geological and geochemical scientific community. (NOTE: This SRM is not a replacement for the fluorspar standard, SRM 79, used primarily for the assay of fluorspar imported for industrial use.) SRM 180 costs $\$ 40.00$ per $120 \mathrm{~g}$ units.

\section{Category 3.56. Minerals, Refractories, Carbides, and Glasses}

SRM 610 - Trace Elements in Glass standards have been issued. These materials consist of a 619 Soda lime glass, doped with some 61 elements at $0.02 \mathrm{ppm}, 1 \mathrm{ppm}, 50 \mathrm{ppm}$ and $500 \mathrm{ppm}$ level. All of these materials are in the form of wafers and are homogeneous when used as integral samples. They are sold as follows:

\begin{tabular}{l|cc|c|c} 
SRM & $\begin{array}{c}\text { Concen- } \\
\text { tration }\end{array}$ & $\begin{array}{c}\text { wafer } \\
\text { thickness }\end{array}$ & $\begin{array}{c}\text { No. of } \\
\text { wafers }\end{array}$ & Cost \\
\hline 610 & $500 \mathrm{ppm}$ & $3 \mathrm{~mm}$ & 6 & $\$ 50.00$ \\
611 & 500 & 1 & 6 & 50.00 \\
612 & 50 & 3 & 6 & 50.00 \\
613 & 50 & 1 & 6 & 50.00 \\
614 & 1 & 3 & 6 & 50.00 \\
615 & 1 & 1 & 6 & 50.00 \\
616 & .02 & 1 & 6 & 50.00 \\
617 & .02 & 3 & 24 & 50.00 \\
618 & set & 1 & 24 & 150.00 \\
619 & set & & &
\end{tabular}

\section{Category 3.61. Nuclear Materials}

SRM 945

SRM 949c

SRM U-0002

SRM U-970
Plutonium Metal Standard Matrix Material has been issued with a Certificate of Analysis. This material has been issued as a matrix material for the preparation of spectroscopy standards. The material costs $\$ 500.00$ per $5 \mathrm{~g}$ units. ${ }^{*}$

Plutonium Metal has been issued. This material is intended as a chemical assay standard for Plutonium. It is priced at $\$ 123.00$ per 0.5 gram unit.*

Uranium oxide-depleted (U-235) has been issued with a Provisional Certificate of Analysis. It is a uranium isotopic standard consisting of highly purified $\mathrm{U}_{3} \mathrm{O}_{8}$, and has a $\mathrm{U}-238$ content of 99.9825 and $\mathrm{U}-235$ content of 0.01733 by weight percent. It is intended for the calibration of mass spectrometers and costs $\$ 58.50$ per $1 \mathrm{~g}$ unit.*

Uranium oxide--enriched (U-235) has been issued with a Provisional Certificate of Analysis. It is a uranium isotopic standard consisting of highly purified $\mathrm{U}_{3} \mathrm{O}_{8}$, and has a $\mathrm{U}-238$ content of 0.5296 and a U-235 content of 97.663 by weight percent. It is intended for the calibration of mass spectrometers and costs $\$ 68.50$ per $1 \mathrm{~g}$ unit.*

*These materials are available only to Atomic Energy Commission contractors and licensees. Order forms and further information may be obtained from the Office of Standard Reference Materials, National Bureau of Standards, Washington, D.C. 20234. 


\section{Category 3.62. Isotopic Reference Standards}

SRM 984

Rubidium Chloride has been issued with a Certificate of Analysis. It is intended as both an assay standard and as an isotopic reference standard. As an assay standard it has a value for $\mathrm{RbCl}$ of 99.9 weight percent; and as an isotopic reference is certified for rubidium with an absolute abundance ratio of ${ }^{85} \mathrm{Rb} /{ }^{57} \mathrm{Rb}$ of 2.593 . This SRM costs $\$ 43.00$ per $1 \mathrm{~g}$ unit.

\section{Category 3.66. Ion Activity Standards}

SRM 186IIc Disodium hydrogen phosphate has been issued with a Certificate. It is intended as $\mathrm{pH}$ standard for use in an admixture only with SRM 186Ic. It costs $\$ 30.00$ per $30 \mathrm{~g}$ unit.

SRM $187 \mathrm{~b}$

Borax has been issued with a Certificate. It is intended as a $\mathrm{pH}$ standard with a $\mathrm{pH}(\mathrm{s})$ value of 9.183 at $25^{\circ} \mathrm{C}$. It costs $\$ 30.00$ per $30 \mathrm{~g}$ unit.

SRM $2201-$ 2202

Sodium Chloride and Potassium Chloride have been certified as ion-selective electrode standard reference materials. These SRM's are the first of a series of materials to be certified for conventional single ionic-activities based on the Stokes-Robinson hydratic theory, which is applicable to ionic strengths greater than 0.1 mole per liter. By means of these SRM's researchers can now standardize their instruments on a common, conventional ionic-activity scale. SRM 2201 costs $\$ 34.00$ per 125 g unit; SRM 2202 costs $\$ 34.00$ per 160 g unit.

\section{Category 4.1. Coating Thickness Standards}

SRM $2301-$ 2308

Gold Coating Thickness Standards (copper clad glass epoxy laminate) have been issued and are certified for weight per unit area (thickness). They are available singly priced at $\$ 66.00$, in sets of two at $\$ 109.00$ and in sets of four at $\$ 182.00$.

\begin{tabular}{l|c|c}
$\begin{array}{l}\text { SRM } \\
\text { Nos. }\end{array}$ & $\begin{array}{c}\text { Nominal Coating Wt. } \\
\left(\mathrm{Mg} / \mathrm{cm}^{2}\right)\end{array}$ & $\begin{array}{c}\text { Nominal Thickness } \\
\text { (micro inches) }\end{array}$ \\
\hline 2301 & 1.5 & 30 \\
2302 & 3.0 & 60 \\
2303 & 6.0 & 120 \\
2304 & 14.0 & 280 \\
2305 & 1.5 and 3.0 & \\
2306 & 3.0 and 6.0 & \\
2307 & 6.0 and 14.0 & \\
2308 & $1.5,3.0,6.0$ and 14.0 &
\end{tabular}

SRM 2311 - Gold Coating Thickness Standards (on copper) have been issued and are Certi2318 fied for weight per unit area (thickness). They are available singly priced at $\$ 66.00$, in sets of two at $\$ 109.00$ and in sets of four at $\$ 182.00$.

\begin{tabular}{l|c|c}
$\begin{array}{c}\text { SRM } \\
\text { Nos. }\end{array}$ & $\begin{array}{c}\text { Nominal Coating Wt. } \\
\left(\mathrm{mg} / \mathrm{cm}^{2}\right)\end{array}$ & $\begin{array}{c}\text { Nominal Thickness } \\
(\text { micro inches })\end{array}$ \\
\hline 2311 & 1.5 & 30 \\
2312 & 3.0 & 60 \\
2313 & 6.0 & 120 \\
2314 & 14.0 & 280 \\
2315 & 1.5 and 3.0 & \\
2316 & 3.0 and 6.0 & \\
2317 & 6.0 and 14.0 & \\
2318 & $1.5,3.0,6.0$ and 14.0 &
\end{tabular}


SRM 2331 2336

SRM 2338 2340

SRM 1475

SRM 1476

SRM 742

SRM 720

SRM 755

SRM 756

SRM 1654

Tin Coating Thickness Standards (on steel) have been issued and are certified for weight per unit area (thickness). They are available singly priced at $\$ 66.00$. SRM 2338 (one each of 2332, 2335) is available at \$109.00; SRM 2339 (one each of $2331,2333,2334,2336$ ) is available at $\$ 182.00$; and SRM 2340 (one each of $2331,2332,2333,2334,2335,2336)$ is available at $\$ 261.00$.

\begin{tabular}{c|c|c} 
SRM No. & $\begin{array}{c}\text { Nominal } \\
\text { Coating Weight } \\
\left(\mathrm{mg} / \mathrm{cm}^{2}\right)\end{array}$ & $\begin{array}{c}\text { Nominal } \\
\text { Thickness } \\
\text { (microinches) }\end{array}$ \\
\hline 2331 & 1.1 & 60 \\
2332 & 2.0 & 110 \\
2333 & 3.0 & 160 \\
2334 & 5.0 & 275 \\
2335 & 12 & 650 \\
2336 & 14 & 750
\end{tabular}

Category 4.5. Molecular Weight Standards

Linear Polyethylene (Whole Polymer) has been issued with a Certificate for molecular weight, limiting viscosity number, melt-flow rate and density. This material is sold in pellet form, priced at $\$ 100.00$ per 50 gram unit.

Branched Polyethylene (Whole Polymer) has been issued with a Certificate for limiting viscosity number and melt-flow rate. The material is sold in pellet form, priced at $\$ 75.00$ per 50 gram unit.

\section{Category 4.21. Freezing and Melting Point Standards}

Aluminum Oxide has been issued with a Certificate as a pyrometric standard with a melting point on the International Practical Temperature Scale (1968) of $2053^{\circ} \mathrm{C}$. This SRM costs $\$ 62.50$ per $10 \mathrm{~g}$ unit.

\section{Category 4.22. Calorimetric Standards}

Synthetic Sapphire $\left(\mathrm{Al}_{2} \mathrm{O}_{3}\right)$ has been issued as a standard reference material for calorimetry. The enthalpy and heat capacity of $99.95+$ percent $\alpha$-alumina are certified over a temperature range from $273.15 \mathrm{~K}$ to $2250 \mathrm{~K}$. SRM 720 costs $\$ 56.00$ per 15 g unit.

Quartz $\left(\mathrm{SiO}_{2}\right)$ powder, prepared from natural quartz, has been Provisionally Certified and issued as a standard reference material for use in thermal analysis. It has a phase transition at approximately $575^{\circ} \mathrm{C}$ and is sold as a powder (100-325 mesh), priced at $\$ 35.00$ per 2 gram unit.

Potassium Nitrate has been Provisionally Certified and issued as a standard reference material for use in thermal analysis. It has a phase transition at approximately $130{ }^{\circ} \mathrm{C}$ and is sold as a powder priced at $\$ 35.00$ per 5 gram unit.

$\alpha$-Quartz for Hydrofluoric Acid Solution Calorimetry has been issued with a Certificate. The certified value for the enthalpy of solution is: $\triangle \mathrm{H}_{\text {SO }} \mathrm{LN}$ $(353.15 \mathrm{~K})$ in HF $(\mathrm{aq}, 24.4 \mathrm{wt} \%)=-2362.2 \pm 1.1 \mathrm{~J} \cdot \mathrm{g}^{-1}$. This SRM costs $\$ 175.00$ per 25 g unit.

\section{Category 4.23. Vapor Pressure Standards}

SRM 746

Cadmium vapor pressure standard has been issued with a Certificate of Analysis. Vapor pressure values for cadmium, $99.999+$ percent pure, are given for the temperature range of 350 to $594 \mathrm{~K}$. This SRM costs $\$ 65.00$ per unit. 
Silver vapor pressure standard has been issued with a Certificate of Analysis. Vapor pressure values for silver, $99.999+$ percent pure, are given for the temperature range of 800 to $1600 \mathrm{~K}$. This SRM costs $\$ 75.00$ per unit.

\section{Category 4.24. Thermal Expansion Standards}

SRM 736

SRM 739

SRM 734

SRM $2001-$ 2004

Copper thermal expansion standard has been issued with a Certificate of Analysis for thermal expansion $(\triangle \mathrm{L} / \mathrm{L})$ as a function of temperature in the range $20-800 \mathrm{~K}$. This material is the first of a series covering the temperature range of 20 to $1900 \mathrm{~K}$. It is available as a $1 / 4$ inch $(6.4 \mathrm{~mm})$ diameter rod in 2,4 , or 6 inch $(51,102$ or $152 \mathrm{~mm})$ lengths. Designated as 736-L1, 736-L2 and 736-L3, respectively, they cost $\$ 71.00, \$ 119.00$ and $\$ 167.00$.

Fused Silica thermal expansion standard has been issued with a Certificate of Analysis for thermal expansion $(\Delta \mathrm{L} / \mathrm{L})$ as a function of temperature in the range 80 to $1000 \mathrm{~K}$. This material is available as a $6.4 \mathrm{~mm}$ diameter rod in 51 , 102, and $152 \mathrm{~mm}$ lengths. Designated 739-L1, 739-L2, and 739-L3, respectively, they cost $\$ 71.00, \$ 119.00$, and $\$ 167.00$.

\section{Category 4.25. Thermal Conductivity Standards}

Electrolytic Iron has been issued with a Certificate for thermal conductivity $(\lambda)$ as a function of temperature in the range 6 to $280 \mathrm{~K}$. This material is the first of a series of thermal conductivity SRM's to be issued. The material is available in three sizes: 734-S is a rod $6.4 \mathrm{~mm}$ in diameter and $305 \mathrm{~mm}$ long, and costs $\$ 75.00$. 734-L1 is a rod $31.8 \mathrm{~mm}$ in diameter and $152 \mathrm{~mm} \mathrm{long}$, and costs $\$ 85.00$. 734-L2 is a rod $31.8 \mathrm{~mm}$ in diameter and $305 \mathrm{~mm}$ long, and costs $\$ 150.00$.

\section{Category 4.45. Reflectance Standards}

Aluminum on Glass have been issued with a Certificate for Specular Spectral Reflectance. Each inirror is certified for near-normal $\left(5^{\circ}\right)$ specular reflectance at wavelengths ranging from 0.2537 to 30 micrometers and corresponding resolved bandwidths from 1.0 to 1800 nanometers. These SRM's cost $\$ 275.00$ ea.

\begin{tabular}{l|l|l} 
SRM No. & Size of blank $(\mathrm{cm})$ & Coated Area $(\mathrm{cm})$ \\
\hline 2001 & $7.6 \times 10.2 \times 1.6$ & $5.1 \times 7.6$ \\
2002 & $3.8 \times 3.8 \times 1.3$ & $2.5 \times 2.5$ \\
2003 & disk: 2.9 diameter $\times 1.0$ thick & entire surface \\
2004 & disk: 2.4 diameter $\times 0.6$ thick & entire surface
\end{tabular}

SRM 2005 - Gold on Glass have been issued with a Certificate for Specular Spectral Re2008 flectance. Each mirror is certified for near-normal $\left(5^{\circ}\right)$ specular reflectance at wavelengths ranging from 0.2537 to 30 micrometers and corresponding resolved bandwidths from 1.0 to 1800 nanometers. These SRM's cost $\$ 275.00$ ea.

\begin{tabular}{l|c|c} 
SRM No. & \multicolumn{1}{|c|}{ Size of blank $(\mathrm{cm})$} & Coated Area $(\mathrm{cm})$ \\
\hline 2005 & $7.6 \times 10.2 \times 1.6$ & $5.1 \times 7.6$ \\
2006 & $3.8 \times 3.8 \times 1.3$ & $2.5 \times 2.5$ \\
2007 & disk: 2.9 diameter $\times 1.0$ thick & entire surface \\
2008 & disk: 2.4 diameter $\times 0.6$ thick & entire surface
\end{tabular}




\section{Category 4.51. Radioactivity Standards}

SRM 4201-B

4211

4213

SRM 4228

SRM 4929-C

SRM 4904D

SRM 485

SRM 493

SRM 1541

SRM 1511

1512

, 1513

Gamma-Ray Point-Sources - have been issued with Certificates. The material is deposited between two layers of polyester tape and mounted on an aluminum annuli $0.8 \mathrm{~cm}$ wide with an outside diameter of $5.5 \mathrm{~cm}$. The material, approximate activity and price are listed below:

\begin{tabular}{l|l|l|r} 
SRM & Material & Activity & Price \\
\hline $4201-\mathrm{B}$ & Niobium 94 & $5 \times 10^{3} \mathrm{ntps}$ & $\$ 151.60$ \\
4211 & Americium 241 & 1 to $6 \times 10^{4}$ & 127.50 \\
$* 4213$ & Americium 241 & 7 to $20 \times 10^{4}$ & 127.50
\end{tabular}

Selenium-75 has been issued with a Certificate. The activity is $2.54 \times 10^{5}$ (3/71) nuclear transformations per second (ntps) per gram of solution. The material is issued in a flame sealed glass ampoule containing approximately 4.6 grams of solution and is priced at $\$ 118.00$.

*This sample can be issued only to those persons who hold specific Byproduct Material License from the AEC. Please attach copy of current license to purchase order.

Iron-55 has been issued with a Certificate. The activity is $7.8 \times 10^{4}(4 / 70)$ nuclear transformations per second (ntps) per gram of solution. The material is issued in a flame sealed glass ampoule containing approximately 3.9 grams of solution and is priced at $\$ 115.00$.

Americium-241 Alpha activity standard has been issued with a Certificate. The standard consists of Americium-241, electroplated onto a 0.010 centimeter thick platinum foil, $1.6 \mathrm{~cm}$ in diameter, which is cemented to a monel disk, $2.5 \mathrm{~cm}$ in diameter, and $0.16 \mathrm{~cm}$ thick. The nominal activity level of this material is $2 \times 10^{3}$ to $5 \times 10^{4}$ nuclear transformations per second (ntps) $(2 / 70)$ and is priced at $\$ 124.00$ per standard.

\section{Category 4.61. Metallurgical Standards}

Austenite in Ferrite primarily for use in calibrating x-ray diffraction equipment, is available in disk form, $21 \mathrm{~mm}$ in diameter and $2.5 \mathrm{~mm}$ thick. This SRM contains four percent austenite, nominally. The actual certified amount is given on each disk (to the nearest 0.1 percent), and is considered accurate to \pm 0.2 percent. SRM 485 costs $\$ 85.00$ per disk.

Spheroidized Iron Carbide $\left(\mathrm{Fe}_{3} \mathrm{C}\right)$ in Ferrite primarily used in calibrating x-ray diffraction equipment, is in wafer form, $29 \mathrm{~mm}$ square and $2.5 \mathrm{~mm}$ thick. The Certificate states that the probability is about 95 percent that the average iron carbide concentration in any wafer is $14.23 \pm 0.30$ percent by volume. SRM 493 costs $\$ 85.00$ per wafer.

\section{Category 4.86. Mossbauer Standards}

Iron foil has been issued with a Certificate of Calibration for Mossbauer spectrometry. SRM 1541 costs $\$ 150.00$ each.

\section{Category 4.87. Permittivity Standards}

Cyclohexane (1511), 1,2-Dichloroethane (1512), and Nitrobenzene (1513) have been issued with Certificates for Dielectric constant at 20,25 and $30^{\circ} \mathrm{C}$. These materials are priced at $\$ 120.00$ per one pint ( 0.47 liter) sample. 

use in calibrating systems for measuring permittivity and related dielectric quantities. Each SRM is individually identified and certified. They cost $\$ 193.00$ per unit. The sizes are:

$1516,38 \mathrm{~mm}$ in diameter and $2.5 \mathrm{~mm}$ thick; $1517,38 \mathrm{~mm}$ in diameter and $5 \mathrm{~mm}$ thick; $1518,51 \mathrm{~mm}$ in diameter and $2.5 \mathrm{~mm}$ thick; $1519,51 \mathrm{~mm}$ in diameter and $5 \mathrm{~mm}$ thick;

\section{Category 5.1. Standard Rubbers and Rubber Compounding Materials}

SRM $373 f$

Benzothiazyl disulfide is now available as a rubber-compounding material. It is issued for use on testing rubber-compounding materials in connection with quality control of raw materials and for the standardization of rubber testing. This SRM costs $\$ 40.00$ per $2 \mathrm{~kg}$ unit.

SRM $374 c$

Tetramethylthiuram Disulfide is now available as a rubber-compounding material. It is issued for use in testing rubber-compounding materials in connection with quality control of raw materials and for the standardization of rubber testing. This SRM costs $\$ 40.00$ per $2 \mathrm{~kg}$ unit.

\section{Category 6.0 Research Materials}

A new class of materials is now being issued to meet the needs of scientists engaged in materials research. Designated Research Materials (RM's), these are in addition to and distinct from the Standard Reference Materials issued by NBS. The distinctions between Research Materials and Standard Reference Materials are in the information supplied with them and purpose for which they are used. Unlike SRM's the RM's are not issued with Certificates of Analysis; rather they are accompanied by a "Report of Investigation," the sole authority of which is the author of the report. A Research Material is intended primarily to further scientific or technical research on that particular material. One of the principal considerations in issuing an RM is to provide homogeneous material so that an investigator in one laboratory can be assured that the material he has is the same as that being investigated in a different laboratory.

RM-1C

Ultra-purity aluminum single crystal cubes ( $1 \mathrm{~cm}$ on a side) are intended for use in studies of a variety of solid state phenomena for which both extreme purity and knowledge of crystallographic orientation are required; e.g., in studies of electron spin resonance, De Haas-Van Alphen effect, cyclotron resonance, etc., and in a variety of studies relating to the Fermi surface and the transport properties of aluminum. RM- $1 \mathrm{C}$ costs $\$ 90.00$ per unit.

RM-1 R

Ultra-purity aluminum polycrystalline rods $(4.2 \mathrm{~mm}$ in diameter and $25.4 \mathrm{~mm}$ long) are intended for use in research on the mechanical and physical properties of extremely pure aluminum: for example, in the determination of resistivity as a function of strain at cryogenic temperatures to facilitate the design of cryogenic magnets or superconductor stabilizing elements. RM-1R costs $\$ 50.00$ per unit. 


\section{Category 7.0. General Materials}

Another new class of materials now being distributed by NBS to meet industry needs is General Materials (GM's). These materials have been standardized either by some Government agency other than NBS, or by some standardsmaking body such as the American Society for Testing and Materials (ASTM), the American National Standards Institute (ANSI), and the Organization for International Standardization (ISO). For this class of materials, NBS acts only as a distribution point and does not participate in the standardization of these materials.

GM-1

Hydrogen in Steel Standards are being distributed by NBS. These standards were produced and certified by The Welding Institute in Cambridge, England. GM- 1 is a set of 15 cylinders, 5 each of $\mathrm{H} 1, \mathrm{H} 2$, and $\mathrm{H} 3$, containing nominally $0.05,0.10$, and $0.20 \mathrm{ml}$ hydrogen, respectively. The cylinders are $6.35 \mathrm{~mm}$ in diameter and about $30 \mathrm{~mm}$ long, weighing approximately 6 grams. GM-1 costs $\$ 86.00$ per set.

GM-2 Hydrogen in Steel Standards are being distributed by NBS. The standards were produced and certified by The Welding Institute in Cambridge, England. GM-2 is a set of 15 cylinders, 5 each of $\mathrm{H} 4, \mathrm{H} 5$, and H6, containing nominally 0.20 , 0.60 , and $1.10 \mathrm{ml}$ hydrogen, respectively. The cylinders are $12.7 \mathrm{~mm}$ in diameter and about $30 \mathrm{~mm}$ long, weighing approximately 22 grams. GM-2 costs $\$ 86.00$ per set.

GM-2007

Attapulgus clay is now being distributed by NBS upon request of the ASTM Committee D-2007. It is an adsorbant type clay, 30 to 60 mesh, having adsorptive characteristics as specified by ASTM D-2007. This GM costs $\$ 143.00$ per $18 \mathrm{~kg}$ (40 lb) unit. 


\section{SECTION III}

\section{MATERIALS OUT OF STOCK}

The materials listed below have gone out of stock since the latest catalog (7/70) was printed. Because funds and facilities are limited, materials that go out of stock are not always renewed; rather, renewals are based on current needs and available funds. If the material you need is not available, please contact the Office of Standard Reference Materials.

\begin{tabular}{|c|c|c|}
\hline $\begin{array}{l}\text { SRM } \\
\text { Nos. }\end{array}$ & Type & Comments \\
\hline $\begin{array}{l}28 \mathrm{a} \\
121 \mathrm{c} \\
132 \mathrm{a} \\
186 \mathrm{IIb} \\
187 \mathrm{a}\end{array}$ & $\begin{array}{l}\text { Iron Ore, Norrie } \\
\text { Steel, Cr18-Ni10(Ti bearing)(SAE 321) } \\
\text { Steel, Mo5-W6-Cr4-V2 } \\
\text { Disodium Hydrogen Phosphate } \\
\text { Borax }\end{array}$ & $\begin{array}{l}\text { Renewed with } 121 \mathrm{~d} \\
\text { To be renewed } \\
\text { Renewed with } 186 \mathrm{II} \text { c } \\
\text { Renewed with } 187 \mathrm{~b}\end{array}$ \\
\hline $\begin{array}{l}373 \mathrm{e} \\
654 \\
727 \\
847 \\
1061 \mathrm{~b}\end{array}$ & $\begin{array}{l}\text { Benzothiazyl disulfide } \\
\text { Ti Alloy, 6Al-4V(B) } \\
\text { Rubidium Chloride } \\
\text { Steel, Cr 24-Ni 13 } \\
\text { Magnesium Cyclohexanebutyrate }\end{array}$ & $\begin{array}{l}\text { Renewed with } 373 \mathrm{f} \\
\text { Renewed with } 654 \mathrm{a} \\
\text { Replaced by } 984 \\
447 \text { and D } 847 \text { have the same composition } \\
\text { Renewed with } 1061 \mathrm{c}\end{array}$ \\
\hline $\begin{array}{l}1079 \mathrm{a} \\
\mathrm{C} 1100 \\
1163 \\
1168 \\
1170\end{array}$ & $\begin{array}{l}\text { Tris(1-phenyl-1,3-butanediono)Iron III } \\
\text { Cartridge Brass A } \\
\text { Low Alloy Steel C } \\
\text { Low Alloy Steel H } \\
\text { Selenium Steel }\end{array}$ & $\begin{array}{l}\text { Renewed with } 1079 \mathrm{~b} \\
\text { Replaced by } 1200 \text { series } \\
\text { Replaced by } 1200 \text { series }\end{array}$ \\
\hline $\begin{array}{l}1174 \mathrm{a} \\
1175 \mathrm{a} \\
1194 \\
1604 \\
4208\end{array}$ & $\begin{array}{l}\text { White Cast Iron (Special 1) } \\
\text { White Cast Iron (Special 2) } \\
\text { A286 High Temperature Alloy } \\
\text { Oxygen in Nitrogen } \\
\text { Mercury 203, Gamma Std. }\end{array}$ & Renewed with $1604 a$ \\
\hline $\begin{array}{l}4225 \\
4924 \\
4929 \mathrm{~B} \\
4995 \mathrm{C} \\
4997 \mathrm{D} \\
4999 \mathrm{D}\end{array}$ & $\begin{array}{l}\text { Tin 113-Indium } 113 \\
\text { Carbon } 14 \text { (water) } \\
\text { Iron } 55 \\
\text { Mercury } 203 \text {, point source } \\
\text { Manganese } 54 \text {, point source } \\
\text { Cerium } 139 \text {, point source }\end{array}$ & Renewed with 4929C \\
\hline
\end{tabular}




\section{SECTION IV}

\section{CHANGES IN PURCHASE PROCEDURE}

\section{ORDERING}

\section{GENERAL}

Orders should be addressed to the Office of Standard Reference Materials, National Bureau of Standards, Washington, D.C. 20234. Telephonic or telegraphic communications should be addressed to the attention of the Office of Standard Reference Materials (Telephone 301-921-2045). Orders should give the amount (number of units), catalog number and name of the standard requested. For example: $150 \mathrm{~g}$ ( 1 unit) of No. $11 \mathrm{~h}$ Basic-Open-Hearth Steel, 0.2 percent C. These materials are distributed only in the units listed.

Acceptance of orders does not imply acceptance of any provision set forth in this order contrary to the policy, practice or regulations of the National Bureau of Standards in the U.S. Government. Prices as listed in this Catalog are subject to change without notice. Price changes when made are first announced in various NBS publications, especially the Technical News Bulletin, and in announcements mailed to users of these materials.

Prices in effect at time of shipment will be billed to the purchaser. No discounts are given on NBS Standard Reference Materials.

To provide better service to users of SRM's our name label files are periodically updated and/or corrected. If your name and address are not correct, please return the mail label portion of the envelope and indicate the corrections. Send it and all other inquiries to:

Office of Standard Reference Materials

National Bureau of Standards

Washington, D.C. 20234

\section{FOREIGN ORDERS}

A. Prepaid orders will be processed, subject to export-import regulations of the United States and country from which order originates, and shipped within 5 days provided export or import license is not required. (See mode of shipment-- Foreign Shipments.) Prepayment may be made by any of the following:

1. UNESCO coupons;

2. Bankers' draft against U.S. bank;

3. Bank to bank transfer on U.S. bank;

4. Letter of credit on a U.S. bank;

5. International Money Order.

All checks, coupons, etc., should be made payable to the National Bureau of Standards and must be in U.S. dollars.

B. Non-prepaid purchase orders from old customers with established credit will be processed within 10 days. Variations in prices and quantities shipped will be noted on invoices. Upon receipt of goods, payment can be made by any of the methods listed under A.

C. Pro-forma service, subject to export-import regulations, may require 60 days or more for processing. Customers are urged to use method A or B whenever possible for fastest service and to supply all necessary import documents and information with their order. Payment may be by any of the means shown under $\mathrm{A}$ above. 


\section{TERMS AND SHIPPING}

\section{DOMESTIC SHIPMENTS}

Shipments of material (except for certain restricted categories, e.g., hydrocarbons, organic sulfur compounds, special nuclear materials, compressed gases and radioactive standards) intended for the United States, Mexico, and Canada are normally shipped prepaid air parcel post (providing that the parcel does not exceed the weight limits as prescribed by Postal Laws and Regulations) unless the purchaser requests a different mode of shipment, in which case the shipment will be sent collect. It is impractical for the Bureau to prepay shipping charges and add this cost to the billing invoice. Hydrocarbons, organic sulfur compounds, compressed gases, rubber compounding materials, radioactive standards and similar materials are shipped express collect.

\section{FOREIGN SHIPMENTS}

A. Small weight shipments over $\$ 100$ in value and prepaid will be shipped by prepaid air parcel post. Shipments exceeding the parcel post weight limit must be handled through an agent (shipping or brokerage firm) located in the U.S. as designated by the purchaser. Parcels will be packed for overseas shipment and forwarded via express collect to the U.S. firm designated as agent.

B. Non-prepaid orders will be shipped by prepaid International Parcel Post, subject to size, weight, and category of material limitations. Any other mode of shipment requested by customer must be paid for by the customer. (Shipments excluded from International Parcel Post for any reason, must be handled through an agent [shipping or brokerage firm] located in the U.S. as designated by the purchaser. These parcels will be packed for overseas shipment and forwarded via express collect to the U.S. firm designated as agent.) 
